\title{
THE GROUP ALGEBRA OF A LOCALLY COMPACT GROUP( ${ }^{(1)}$
}

\author{
BY \\ I. E. SEGAL
}

Introduction. This paper concerns the theory of ideals in the algebra (called the group algebra) of all complex-valued functions on a locally compact (abbreviated to LC) group which are integrable with respect to Haar measure, multiplication being defined as convolution. It is proved that the group algebra of a group which is either LC abelian or compact is semisimple, an algebra being called semi-simple in case the intersection of all regular maximal ideals is the null ideal (a regular ideal is defined as one modulo which the algebra has an identity). This extends the theorem that the group algebra of a finite group is semi-simple. A weaker kind of semi-simplicity is proved in the case of a general LC group, an algebra being called weakly semi-simple when both the intersection of all regular maximal right ideals is the null ideal and the same for left ideals. (In the case of a finite-dimensional algebra these concepts of semi-simplicity are equivalent to that of Wedderburn, and in the case of a commutative Banach algebra with an identity - which Gelfand designates as a "normed ring"-are equivalent to Gelfand's concept of the vanishing of the radical of the algebra.)

It is shown that an ideal in a (strongly) semi-simple algebra can be resolved into regular ideals in an approximate fashion, the approximation being in terms of a topology which is (algebraically) introduced into the family of all regular maximal ideals. This topologized family (which is called the spectrum of the algebra, this term being suggested by the case of the algebra generated by a linear operator) is determined in explicit fashion for the group algebra of a group which is either LC abelian or compact, and partially determined when the group is discrete. Specifically, in the case of a group which is respectively either LC abelian, compact, or discrete, the spectrum of the group algebra is homeomorphic with the group of continuous characters, discrete or compact. A closed ideal in a group algebra is the kernel of a kind of extension from the group to the group algebra of a bounded strongly continuous representation of the group by linear operators on a Banach space. If the

Presented to the Society, November 2, 1946; received by the editors February 9, 1946.

(1) This paper is an extension of a part of the author's doctoral dissertation which was presented to the faculty of Yale University in April, 1940. A summary of most of the paper was published in [30], and Bull. Amer. Math. Soc. Abstract 46-7-366 is pertinent. The "group ring" considered in [30] consists of the present group algebra modified, in case it does not contain an identity, by the adjunction of an identity. Some of the material in the paper was worked on while the author was at Harvard University. Numbers in brackets refer to the bibliography at the end of the paper. 
ideal is regular and maximal, the representation is irreducible; and for a group which is either LC abelian or compact, the ideal is regular and maximal if and only if the representation is finite-dimensional and irreducible (in the case of a LC abelian group this correspondence implements the homeomorphism mentioned above between the spectrum of the group algebra and the group of continuous characters).

These theorems have applications, which are partly new results and partly great simplifications in the proofs of known results, to the theory of bounded representations of LC groups and to harmonic analysis. A LC group has a complete set of strongly continuous irreducible representations by bounded operators on Banach spaces (irreducible in the sense that there exists no nontrivial invariant subspace). (Gelfand and Raikov [16] have proved that the same theorem is true for representations by unitary operators on Hilbert spaces, with a weaker kind of irreducibility, by a method unrelated to ours.) The Peter-Weyl-von Neumann approximation theorem for almost periodic functions on groups [27] is shown to be essentially equivalent to the fact that the group algebra of a compact group is semi-simple. A theorem of Tauberian type is obtained which considerably generalizes Wiener's general Tauberian theorem [37], specifically as regards: (a) the set of zeros of the Fourier transform of the kernel (the null set in Wiener's case, and arbitrary here); (b) the space of the kernel (either the group algebra of the reals or a certain ideal in that algebra, in Wiener's case, and here one of a general class of ideals in the group algebra); (c) the group over which the functions are defined (the additive group of the reals in Wiener's case, and here an arbitrary LC abelian group). (Wiener's theorem has also been extended as regards (a) and (b) by Pitt [29], in a series of theorems of which some are very special cases of our results.) A similar generalization is made of Wiener's results about the span of the translations of elements of Lebesgue spaces over the reals. The basic case of a theorem about the existence of analytic functions of absolutely convergent Fourier-Stieltjes transforms which was proved independently by Beurling [4] and by Cameron and Wiener [6] is extended from the reals to arbitrary LC abelian groups, and partially extended to compact (not necessarily abelian) groups.

We note at this point that many of our results, in so far as they refer to the group algebra of a LC group which is abelian, were independently obtained by Gelfand and Raikov. Gelfand's paper [10] investigating Banach algebras with identities, which was one of the keys to these results, was known to the author fairly early in his work. On the other hand, the later papers, [11] by Gelfand and [15] by Gelfand and Raikov, in which it was recognized that the methods introduced in [10] could be fruitfully applied to harmonic analysis, and [17] by Gelfand and Silov, in which the set of maximal ideals in a commutative algebra with an identity is given various topologies, one of which is identical with the topology we employ in the spectrum, were not 
known to the author until after his work on the abelian case was complete $\left({ }^{2}\right)$. The spectral resolution of ideals in the algebra appears, however, to be a previously unrecognized development of importance for harmonic analysis.

The type of decomposition that we consider for ideals in group algebras is one as an intersection of regular maximal ideals, which differs from the type usually considered for algebras, this being one as a direct sum of minimal ideals. The reason for the difference is that the latter type of decomposition cannot be utilized in the case of group algebras, because of the fact that minimal ideals do not exist, in general (for example, the group algebra of the reals does not contain any minimal ideals). On the other hand, given a regular ideal in an arbitrary algebra, there exists a regular maximal ideal containing it, and it is therefore natural to expect that a decomposition into regular maximal ideals should be more generally valid than one into minimal ideals. As a matter of fact, a number of fairly recent investigations of the theory of representations of various types of rings (none of which were assumed to satisfy any finite chain condition-a kind of assumption which is rarely satisfied in the case of a ring originating in analysis) have likewise considered the regular maximal ideals as the basic constituents of the algebra. In this category are notably the investigations of: Stone into Boolean rings; $\mathrm{McCoy}$ and Montgomery into generalized Boolean rings; and Gelfand and his associates into commutative Banach algebras with identities. The work of these authors is similar to ours in two additional important respects. In the first place, they are also strongly concerned with the semi-simplicity of the rings which they consider (semi-simplicity for rings being defined in the same way as for algebras)-the main conclusions of Stone's paper [33] and McCoy and Montgomery's paper [24] being respectively essentially that a Boolean ring is semi-simple, and the same for generalized Boolean rings. Secondly, some of these authors have introduced, in connection with the rings they consider, the space which we have designated the spectrum (Stone [34]; Gelfand and Silov [17]) $\left.{ }^{3}\right)$. Our point of view differs from that of these authors chiefly in our somewhat greater interest in decompositions of general ideals (as well as null ideals) $\left.{ }^{4}\right)$. In the case of a group algebra, decompositions of closed ideals

(2) These results, with the exception of the ideal spectral resolution, were presented in Bull. Amer. Math. Soc. Abstract 46-7-366.

(3) Some algebraic systems other than rings have been investigated from essentially the same viewpoint: distributive lattices by $\mathrm{H}$. Wallman and $M$-spaces by $\mathrm{S}$. Kakutani and $\mathrm{M}$. and S. Krein. The principal conclusion of each of these authors can be stated as the theorem that each system is semi-simple, in the same sense as in the case of algebras, ideals being suitably defined; and each of these authors topologized the set of regular maximal ideals in a way which resulted in a topology analogous to that on the spectrum of an algebra.

(4) We mention that Gelfand [12] has considered decompositions of general ideals in Banach algebras satisfying certain special conditions; whether any nontrivial group algebras satisfy these conditions we do not know. In the case of a generalized Boolean ring, an exact decomposition for general ideals is an easy consequence of semi-simplicity, following from the evident fact that a homomorphic map of such a ring is a ring of the same type. 
into regular maximal ideals are closely connected (by virtue of the relation between ideals and group representations) with decompositions of group representations into irreducible representations.

A point of technical interest is that by omitting the usual assumption that the algebra under consideration has an identity, a more natural definition of group algebra can be given, and there results from this definition a more suggestive kind of spectrum for the group algebra (the spectrum of an algebra with an identity being necessarily compact). It is this (possible) lack of an identity which makes it necessary at times to consider regular (or regular maximal) ideals rather than fully general (or general maximal) ideals.

The analytical basis of our work consists mainly of various parts of the theory of operators on a Banach space together with the same theory for a Hilbert space. Our utilization of Hilbert space theory rests on the observation that the group algebra is (algebraically) isomorphic with an algebra of bounded operators on the space $L_{2}$ of complex-valued functions on the group which are square-integrable with respect to Haar measure; an element $x$ of the group algebra corresponds to the operator taking $y$ into $x y, y$ being a general element of $L_{2}$ and $x y$ being the convolution of $x$ with $y$. In its utilization of Hilbert space methods and consideration of noncommutative algebras our work is similar to an interesting paper of Ambrose [3], but differs from this paper considerably because Ambrose investigated direct-sum decomposition into minimal ideals. His structure theorems when applied to the case of an algebra essentially equivalent to the group algebra of a compact group (in which minimal ideals exist) imply the semi-simplicity of this former algebra (from which the semi-simplicity of the group algebra follows easily), as well as other facts about compact groups.

There remain open a number of natural questions about the ideal theory of a general group algebra. Specifically: (a) s the group algebra of a (general) LC group semi-simple? (b) Is every bounded strongly continuous representation of a LC group by operators on a Banach space equivalent to a strongly continuous representation by unitary operators on a Hilbert space? More generally, we inquire about the topology on the spectrum of the group algebra, and the extent of the duality between the spectrum and the group. (Is an analogue of the Plancherel theory valid for general LC groups?) We hope that the present paper may suggest methods of dealing with these questions.

This present paper is an extension of part of a doctoral thesis whose topic was suggested by Einar Hille. Specifically, Hille suggested a continuation of the investigations by himself and Tamarkin $[18,20,21,22,23]$ of the ideal theory of the algebra of Laplace-Stieltjes transforms absolutely convergent in a fixed half-plane, this subject being closely related to the ideal theory of the group algebra of the reals. For conceptual clarification and for other reasons, an investigation of the ideal theory of the group algebra of a general LC abelian group was of interest. In the case of a compact abelian group, the 
Riesz-Hildebrandt theory of completely continuous operators was an effective tool in proving the semi-simplicity of the group algebra and in showing that the obvious necessary condition that an element of the group algebra have an adverse (which is equivalent to the condition that an element of the group algebra with an identity adjoined have an inverse-see below for a definition of the adverse, which essentially takes the place of the inverse in an algebra which is not assumed to have an identity) is also sufficient. (Hille and Tamarkin [19] had previously proved the latter theorem in the case of the group algebra of the reals modulo unity.) It was clear that the same theorem could be proved for the group algebra of a compact group which was not necessarily abelian, by slightly modified methods. In a conversation with B. J. Pettis about our thesis, Pettis mentioned Gelfand's paper [10] as somewhat concerned with the ideal theory of Banach algebras. In this paper (which followed papers by Gelfand and others about algebras of bounded continuous functions on topological spaces) it was shown (among other results) how the units in a commutative Banach algebra with an identity could be determined once the maximal ideals were known. On the other hand, the existence of maximal ideals in such an algebra was proved by transfinite induction. It was almost too much to hope that it could be proved (without essentially determining the units in the process) that there existed no maximal ideals in the group algebra of a LC abelian group except those which were apparent. It was plain that such a proof would lead to great simplifications in important aspects of harmonic analysis (in particular to a much simplified proof of Wiener and Pitt's theorem in [29a] about the existence of adverses in the group algebra of the reals). Fortunately such a proof was found, and the theorem about the existence of adverses which had previously been proved for the group algebra of a compact group followed easily for LC abelian groups. The fact that the semi-simplicity of the group algebra of a compact group essentially implied the approximaton theorem for almost periodic functions on groups, together with the foregoing results for the case of a LC abelian group, naturally suggested that an investigation of general group algebras, with especial regard to semi-simplicity, might be valuable $\left(^{b}\right)$.

In Part I semi-simplicity of group algebras is considered, and a relation between ideals in a group algebra and representations of the group is established. The existence of Haar measure (and its measure-theoretic consequences, as exposed in Chapters II and III of [35]) is the only special premise used. In Part II a spectral resolution for ideals in a general algebra is obtained, and applied to ideals in the group algebra of a group which is either

(s) In 1941 war work (from 1943 in the form of military service) interrupted our investigation of group algebras, and its continued pressure was largely responsible for the great delay in the submission for publication of the material then on hand. In preparing this material for publication we have made various refinements, the chief one being an increased generality for the ideal spectral resolution. 
LC abelian or compact. The topology on the spectrum of such a group algebra is considered, utilizing the analogues of the Plancherel theory for a group which is either LC abelian or compact (see, for example, Chapters V and VI of [35]). Part III covers applications to harmonic analysis on groups: Tauberian theorems, determination of the span of the translations of functions, analytic functions on group algebras, and a new approach to the theory of almost periodic functions on groups.

We are deeply indebted to Warren Ambrose for many interesting conversations about locally compact groups, which led to extensive simplifications in the development of our theory.

\section{Part I. Semi-simplicity of Group algebras}

1. Definitions. In Part I our main object is to investigate group algebras with regard to semi-simplicity, the definitions of these terms being given in the present section.

Definition 1.1. A (right, left, two-sided) ideal in an algebra is called: regular if there exists $\left({ }^{6}\right)$ an element of the algebra that is a (left, right, twosided) identity modulo the ideal ( $e$ is a left identity modulo an ideal if $e x-x$ is in the ideal for every $x$ in the algebra); maximal if it is proper and contained in no other proper ideal. An ideal is a two-sided ideal, unless otherwise specified.

Definition 1.2. An algebra is called (strongly) semi-simple if the intersection of all regular maximal ideals is the null ideal. It is called weakly semisimple if both: (a) the intersection of all regular maximal right ideals is the null ideal; (b) the same is true for left ideals.

Definition 1.3. The group algebra $A$ of a LC group $G$ is the topological algebra consisting of all complex-valued functions on the group which are integrable with respect to (left-translation invariant) Haar measure, the topology being obtained by norming a general function with the integral of its absolute value, and algebraic operations being defined as follows:

$$
(\alpha f)(x)=\alpha f(x), \quad(f+g)(x)=f(x)+g(x), \quad(f g)(x)=\int_{G} f(y) g\left(y^{-1} x\right) d y .
$$

Here, $f$ and $g$ are general elements of $A, x$ is a general element of $G, \alpha$ is a general complex number, and $d y$ refers to Haar measure $\left({ }^{7}\right)$.

(8) Such ideals should strictly be called "(right, left or two-sided) regular," but in order to avoid circumlocution the qualifying phrase is omitted, it being clear from the context what kind of regularity is meant. It may clarify this notion to explain that a two-sided ideal $I$ in an algebra $A$ is regular if and only if $I$ can be extended to an ideal $\bar{I}$ of the algebra $\bar{A}, \bar{A}$ being obtained from $A$ by adjoining a unit (in case it does not already have one), and the extension being in the sense that $\bar{I} \cap A=I, \bar{I} \cup A=\bar{A}$.

${ }^{(7)}$ There exist other interesting algebras associated with LC groups which generalize the group algebra of a finite group, notably the weak closure $R$ of the algebra generated by the unitary operators, on the space of square-summable complex-valued functions on the group $G$, 
2. Existence of adverses. In this section it is shown that whether the adverse $\left({ }^{8}\right)$ of an element of a general algebra exists essentially depends largely on whether the adverse exists modulo regular maximal ideals $\left({ }^{9}\right)$, and that the same is true as regards the existence of a right adverse in relation to the regular maximal right ideals.

Definition 1.4. An element $y$ of an algebra is called a left (right) adverse of an element $x$ of the algebra in case $x+y=y x(x+y=x y)$, and is called simply an adverse in case it is both a left and a right adverse. An adverse of $x$ is denoted $x^{A}$ (the next theorem shows that an element can have at most one adverse).

corresponding to left translations on the group. (In the case of a discrete group this algebra has been investigated by Murray and von Neumann $[25,26]$.) So far as the author knows, the first generalization of this type was made by Peter and Weyl [28], who considered the numericalvalued continuous functions on a compact group as a kind of group algebra, with multiplication defined as convolution. In the case of a compact group it happens that nearly all of the usual numerical function spaces are algebras with respect to convolution (this being due essentially to the fact that the measure of the group is then finite); the algebra of square-summable functions on a compact group has been investigated by Ambrose [3]. But, in general, $A$ is the only one of the usual function spaces which is an algebra.

The ideal theory of $R$ is closely related to the theory of unitary representations of $G$, and is in some respects simpler than the ideal theory of $A$. On the other hand, $A$ has the significant advantages that: (a) rather general representations of $G$ correspond in a simple fashion to representations of $A$ (whether they correspond in a natural way to representations of $R$ is an open question); (b) the ideal theory of $A$ is fundamental in important aspects of harmonic analysis on the reals; (c) in the case of a LC abelian group the spectrum of $A$ is, rather suggestively, the continuous character group (the spectrum of $R$ is a compact space of a rather complicated sort, being non-separable in case $G$ is the reals; in general, for a LC abelian group, $R$ is isomorphic with the algebra of measurable, essentially-bounded, numerical functions on the continuous character group, with multiplication defined in pointwise fashion). We note that $A$ is algebraically isomorphic to a weakly dense sub-algebra of $R$.

It would be fundamentally awkward to consider the real-valued integrable functions on the group as the group algebra, for a variety of reasons. For example, the quotient algebra of this real algebra over a LC abelian group modulo a regular maximal ideal is isomorphic with the algebra of complex numbers (over the real field).

(8) We prefer the term "adverse" to the more usual term "quasi-inverse" (and the quasiinverse is the negative of the adverse). Consideration of adverses is necessary because a group algebra does not in general have an identity (see Theorem 1.10). In the case of an algebra with an identity $i$, the inverse can be used instead of the adverse, the (right, left, two-sided) adverse of an element $x$ existing if and only if the (left, right, two-sided) inverse of $x-i$ exists. We prefer not to adjoin an identity to the group algebra, partly because it complicates the spectrum of the algebra, and partly because it is the ideal theory of the algebra without the identity which is directly pertinent for applications to Tauberian theorems and spanning of translations.

(9) Not entirely, however. It is clear from Calkin's theorem [5] that the ideal of completely continuous operators is the only maximal ideal in the (Banach) algebra of bounded operators on Hilbert space (defining the Banach norm of an operator to be its bound), that there exist elements in this algebra which have an adverse modulo every maximal ideal without having an adverse (in the large) - namely the identity minus a completely continuous operator for which unity is a proper value. 
THEOREM 1.1. If an element of an algebra has both a right and a left adverse, then it has a (full) adverse, and this adverse is unique.

The proof is very analogous to that for inverses.

Theorem 1.2. A proper regular (right, two-sided) ideal in an algebra is contained in some regular maximal (right, two-sided) ideal.

Putting $I$ for the (right, two-sided) ideal and $e$ for an identity modulo $I$, then by Zorn's lemma there exists a (right, two-sided) ideal $M$ which is maximal with respect to not containing $e$. It is easy to verify that $M$ is a regular maximal (right, two-sided) ideal.

THEOREM 1.3. A NASC $\left({ }^{10}\right)$ that the (right, full) adverse of an (element, element of the center) of an algebra exist is that it exist modulo every regular (right, two-sided) ideal in the algebra.

The condition is obviously necessary. Now suppose that $x$ is an (element, element of the center) of the algebra $A$, and that $x$ has an adverse modulo every regular maximal (right, two-sided) ideal, but no (right, full) adverse. Setting $I=[x y-y \mid y \in A]\left({ }^{11}\right)$, it is easy to verify that $I$ is a regular (right, two-sided) ideal ( $x$ being an identity modulo $I$ ) and that $I$ is contained in no regular maximal (right, two-sided) ideal. By Theorem 1.2, $I=A$, implying $x y-y=x$ for some $y$, a contradiction.

3 . Concerning the semi-simplicity of general group algebras. The existence of the adverse of an element of a group algebra implies, it will be seen the existence of the inverse of an associated bounded linear operator on a Hilbert space. Consideration of the existence of this inverse, in the light of the spectral theorem for self-adjoint operators, yields a restriction on the existence of adverses; this restriction, utilizing the results of the preceding section, is used to show that a group algebra is weakly semi-simple, and also that the intersection of the radical (see following definition) and the center of a group algebra is the null ideal. These facts are proved in the present section; the latter fact is used in the following section to show that the group algebra of the direct product of a LC abelian and compact group is semi-simple.

DEFINITION 1.5. The radical of an algebra is the intersection of all regular maximal ideals in the algebra.

Definition 1.6. For a complex-valued function $f(\cdot)$ on a LC group, $f^{*}(\cdot)$ is defined by: $f^{*}(x)=\rho(x)\left(f\left(x^{-1}\right)\right)^{c}\left({ }^{12}\right)$, where $d x^{-1}=\rho(x) d x\left({ }^{13}\right)$.

(10) NASC is used as an abbreviation for "necessary and sufficient condition."

(11) The notation $[f(x) \mid(x$ has the property $\mathrm{P})]$, where $f(x)$ is a function of $x$, is used to denote the range of values of $f(x)$ for all values of $x$ having the property $\mathrm{P}$.

(12) The notation $(\cdot)^{c}$ is used to denote either the complex conjugate of the expression in parentheses or the topological closure, depending on the context.

(13) Compare [35, p. 40] (where the notation $\Delta\left(x^{-1}\right)$ is used for $\rho(s)$ ). 
This definition of $f^{*}(\cdot)$ is used instead of the more usual $\left(f\left(x^{-1}\right)\right)^{c}$ for reasons which are plain from Theorem 1.4.

THEOREM 1.4. If $f$ is in the group algebra of the LC group $G$, then so is $f^{*}$. Putting $T_{f}$ for the bounded operator on $L_{2}(G)$ defined by $\left({ }^{14}\right)$

$$
T_{f} g=f g, \quad g \in L_{2}(G),
$$

then the adjoint of $T_{f}$ is $T_{f^{*}}$.

Using Fubini's theorem, Theorem 1.4 is easily verified.

The following trivial corollary embodies an important algebraic aspect of group algebras, which allows immediate translation of a theorem about right ideals in to a theorem about left ideals.

COROLLARY 1.4.1. If $f$ and $\xi$ are elements of the group algebra of a LC group and $\alpha$ is a complex number, then: $(f g)^{*}=g^{*} f^{*},(f+g)^{*}=f^{*}+g^{*}, f^{* *}=f$, and $(\alpha f)^{*}=\alpha^{c} f^{*}$.

THEOREM 1.5. The group algebra of a LC group is weakly semi-simple, and the intersection of its radical with its center is the null ideal.

The proof is indirect. Assume that $h$ is in the intersection of (all regular maximal right ideals, the radical and the center). Putting $f=h h^{*}$, plainly $f$ is in the intersection (of all regular maximal right ideals and all regular maximal left ideals, the radical and the center). By Theorems 1.1 and 1.3 and the fact that 0 has an adverse, $\alpha f$ has an adverse for every complex number $\alpha$. Defining $T, U_{\alpha}$ and $I$ by:

$$
T g=f g, \quad U_{\alpha} g=(\alpha f)^{A} g, \quad I g=g,
$$

where $g$ is a general element of $L_{2}$ on the group, then clearly these are all bounded operators, and, by Theorem 1.4, $T$ is self-adjoint. It is easy to verify that:

$$
\left(I-U_{\alpha}\right)(I-\alpha T)=I=(I-\alpha T)\left(I-U_{\alpha}\right) .
$$

It follows that the spectrum of $T$ consists only of zero; and therefore, by the spectral theorem for a self-adjoint operator, $T=0$. Hence $f=0$ and $h=0$, concluding the proof $\left({ }^{15}\right)$.

4. The group algebra of the direct product of a LC abelian group and a compact group. In this section it is proved that the group algebra of the direct product of a LC abelian group and a compact group is semi-simple.

(14) The convolution of an element of $L_{1}$ with an element of $L_{2}$ is necessarily an element of $L_{2}$; see $[35$, p. 50$]$.

(15) We have incidentally proved, clearly, that any self-adjoint algebra of bounded operators on a Hilbert space is weakly semi-simple.

It is interesting that this method also shows that a Boolean ring is weakly semi-simple, it being easy to verify that 0 is the only element which has either a right or a left adverse. Stone's representation theorem for a Boolean ring follows. 
The proof proceeds essentially by showing that every non-null closed characteristic ideal in such a group algebra meets the center in a nonzero element, and that the radical of a group algebra is closed, and then applying Theorem 1.5. The theory of completely continuous operators is not used.

THEOREM 1.6. In a topological algebra with the property that a (right, full) adverse of every element in some neighborhood of zero exists, every regular maximal (right, two-sided) ideal is closed.

Putting $A$ for an algebra with the property stated in the theorem, and $M$ for a regular maximal (right, two-sided) ideal, it follows easily that $\bar{M}$ (denoting the closure of a set by superposing a bar) is a (right, two-sided) ideal. It is evidently sufficient to show that $\bar{M}=A$ is false. Employing an indirect argument, suppose that $\bar{M}$ is $A$. Putting $e$ for a (left, two-sided) identity modulo $M$, it is clear that there exists an element of $M, m$, such that the (right, full) adverse of $e-m$ exists. Putting $z$ for this (right, full) adverse, then the defining equation of $z$,

$$
(e-m) z=e-m+z,
$$

yields a contradiction when reduced modulo $M$.

Definition 1.7. A Banach algebra (or simply $B$-algebra) is an algebra whose linear space is identical with that of a Banach space, and such that $\|x y\| \leqq\|x\| \cdot\|y\|$ for general elements of the algebra $x$ and $y$.

Corollary 1.6.1. A regular maximal right or two-sided ideal in a $B$-algebra is closed.

It is easy to verify that if $\dot{x}$ is an element of a $B$-algebra with $\|x\|<1$, then $y=-\sum_{n-1}^{\infty} x^{n}$ is absolutely convergent and $x y=y x=x+y$. Therefore the hypothesis of Theorem 1.6 is satisfied.

THEOREM 1.7. The group algebra of the direct product of a LC abelian and compact group is semi-simple.

Let $G$ be the direct product group and $C$ the compact group. Putting $N$ for the radical of the group algebra, and noting that a group algebra is a $B$-algebra (using the $L_{1}$ norm, as previously), it follows that $N$ is closed. Now suppose $h \in N$, let $k$ be a general element of the intersection of $L_{1}(G)$ and $L_{2}(G)$, and put

$$
d=h k, \quad g=d d^{*}, \quad f(\cdot)=\int_{C} g_{a}(\cdot) d a,
$$

where $g_{a}(x)=(g)\left(a^{-1} x a\right)$ (taking the integral in the Bochner-Dunford-Hildebrandt sense) (16) and where $a^{-1} x a$ is defined for $a \in C$ by identifying $C$ with the subgroup of $G:\left[\left(c, e^{\prime}\right) \mid c \in C\right]$, where $e^{\prime}$ is the identity of the abelian group.

(16) See [9] for an account of this integral; we shall refer to it as the $\mathrm{BDH}$ integral. 
From the obvious fact that the radical is invariant under automorphisms of the group algebra (and hence, in particular, the automorphisms $r(x) \rightarrow r\left(a^{-1} x a\right)$, where $r(\cdot)$ is a general element of the group algebra) it follows that $f(\cdot) \in N$. Also, it is clear that $f\left(a^{-1} x a\right)=f(x)$ for every $a \in G$, from which it follows that $f(\cdot)$ is in the center of the group algebra. Hence (by Theorem 1.5) $f(\cdot)=0$. To conclude the proof it is necessary to show that this last result implies $h=0$. Now $g$ is plainly a continuous function, and therefore the integral defining $f(\cdot)$ exists in the pointwise Lebesgue sense; thus $f(e)=m \int_{G}|d(x)|^{2} d x$, where $m$ is the measure of $C$ and $e$ is the group identity. Therefore $d=0$, which implies $h=0$ by virtue of the general character of $k\left({ }^{17}\right)$.

5. Bounded group representations. In the present section it is shown that a closed left ideal in the group algebra of a LC group corresponds in a natural fashion to a bounded strongly continuous representation of the group by linear operators on a $B$-space (such a representation is called a $B$-representation). Moreover, in case the ideal is maximal, the representation is irreducible, in the sense that the only invariant subspaces are the trivial ones- 0 and the entire space. It then follows, utilizing the weak semi-simplicity of the group algebra, that a LC group has a complete set of irreducible $B$-representations: In the special case of a group which is either abelian or compact, it is shown that an ideal is regular and maximal if and only if a corresponding group representation is finite-dimensional, bounded, continuous, and irreducible.

DEFINITION 1.8. A Banach representation (or simply $B$-representation) of a LC group $G$ on a Banach space $B$ is a function $T(\cdot)$ on $G$ to the algebra of bounded linear operators on $B$ such that: (a) $T(\cdot)$ is continuous in the strong operator topology; (b) $T(a b)=T(a) T(b)$ for $a, b \in G$; (c) $\|T(a)\|$ is bounded for $a \in G$; (d) $T(i)$ is the identity operator, $i$ being the group identity. Such a representation is called irreducible if there exists no proper subspace of $B$ which is invariant under $T(a)$, for all $a \in G$, except the null space. The mapping $f(\cdot) \rightarrow \int_{G} T(x) f(x) d x$, where $f(\cdot)$ is a general element of the group algebra $A$, and the integral is defined to be the operator taking the general point $b$ of $B$ into the BDH integral $\int_{G} f(x)(T(x) b) d x$, is called the extension of $T(\cdot)$ to $A$. A family of $B$-representations of $G$ is called complete if the identity is the only element of the group which is mapped into the identity operator by every representation in the family.

In connection with this definition it should be mentioned that it is easy to verify that the extension of a $B$-representation of a LC group is a continuous homomorphism of the group algebra.

TheOREM 1.8. Let $K$ be a closed left ideal in the group algebra $A$ of the LC group $G$. Then there exists a $B$-representation of $G$ on the Banach quotient space

(17) There exist groups whose algebras are semi-simple and which are not of the type considered in Theorem 1.7. An example is a free group with any number of generators, in the discrete topology. 
$A-K$, the kernel $\left.{ }^{18}\right)$ of the extension of this representation being (the ideal) $[x \mid x A \subset K]$. In case $K$ is a maximal left ideal this representation is irreducible.

For $u \in A$ and $a \in G$, define $u_{a}$ by $u_{a}(x)=u\left(a^{-1} x\right)$. Defining $T_{a}$ on, $A-K$ by $T_{a}(u+K)=u_{a}+K$, it is clear that in order for this definition to be valid (for $T_{a}$ to be single-valued), it is sufficient that $u_{a}$ belong to $K$ whenever $u$ does. To show that this is the case, recall that $[35, \mathrm{p} .52]$ for any $u \in A$ there exists a sequence $v_{n} \in A$ such that $v_{n} u \rightarrow u$, as $n \rightarrow \infty$. It is easy to verify that the relation $v_{n} u \rightarrow u$ implies $\left(v_{n}\right)_{a} u \rightarrow u_{a}$. Now if $u \in K,\left(v_{n}\right)_{a} u \in K$ because $K$ is a left ideal, and hence, because $K$ is closed, $u_{a} \in K$, thus $T_{a}$ is well-defined. It is easy to verify that the mapping $a \rightarrow T_{a}$ is a $B$-representation of $G$. It follows readily from the theory of the BDH integral that the extension of this representation to $A$ is the mapping $x \rightarrow S_{x}$, where $S_{x}(u+K)=x u+K\left({ }^{19}\right)$. It is plain that the kernel of this homomorphism is as stated. A subspace of $A-K$ which is invariant under the group representation is also invariant under its extension, using the fact that a BDH integral is in the span of the range of values of the integrand. It follows that if $K$ is maximal, the representation is irreducible.

Corollary 1.8.1. A LC group has a complete set of irreducible Banach representations.

Designate the $B$-representation of the LC group $G$ corresponding to the regular maximal left ideal $K$ (which is closed by Corollary 1.6.1) by $T_{a}(K)$. Now if $T_{a}(K)=T_{i}(K)$, then clearly $u_{a}-u \in K$ for every $u \in A$; and if $a$ is represented by the identity operator by all such representations, then by the weak semi-simplicity of the group algebra, $u_{a}=u$ for every $u \in A$, which implies $a=i$.

Definition 1.9. A $B$-algebra $A$ with the property that the operators $x \rightarrow u x$ and $x \rightarrow x u(x, u \in A)$ on $A$ to $A$ are completely continuous (abbreviated to $\mathrm{CC}$ ) is called a $\mathrm{CC}$ algebra.

THEOREM 1.9. Let $G$ be a group which is either LC abelian or compact. Then every regular maximal ideal in the group algebra is the kernel of the extension of a bounded, continuous, irreducible representation of $G$ by finite-dimensional matrices. Conversely, the kernel of the extension of such a representation is regular and maximal, and the kernels of the extensions of two such representations

(18) The kernel of a homomorphism of an algebra is the collection of elements which are mapped into zero by the homomorphism. If $Y$ is a closed linear subspace of a Banach space $X$ the Banach quotient space $X-Y$ is the algebraic quotient space with the norm $\|x+Y\|$ $=\inf _{y} \in Y\|x+y\|$. It is known that a Banach quotient space is a Banach space.

(i9) More generally, a strongly continuous homomorphism of the group algebra onto the algebra of bounded operators on a $B$-space, $B$, is the extension of a $B$-representation of $G$ on $B$, in case the kernel is regular. Putting $e$ for an identity modulo the kernel and $S(x)$ for the operator into which $x(\in A)$ maps, it can be shown that $a \rightarrow S\left(e_{a}\right)$ is a $B$-representation of $G$, and that the extension of this representation is $x \rightarrow S(x)$. 
are the same if and only if the representations are similar $\left({ }^{20}\right)$.

First consider the case of a compact group. Then $u x$ can be written as the $\mathrm{BDH}$ integral $\int_{G} u^{a} x(a) d a$, where $u^{a}$ is an element of $A$ for each $a$, defined by $u^{a}(t)=u\left(t a^{-1}\right)$. Since the operation $a \rightarrow u^{a}$ is continuous on $G$ to $A[35$, p. 41] the set $\left[u^{a} \mid a \in G\right]$ is compact. It follows, from a theorem of Dunford and Pettis about BDH integrals [9, Theorem 3.1.10] that the operation $x \rightarrow u x$ on $A$ to $A$ is $\mathrm{CC}$. In the same way it can be shown that $x \rightarrow x u$ is $\mathrm{CC}$, and therefor $A$ is a $\mathrm{CC}$ algebra. It is easy to verify that a $B$-quotient algebra( $\left.{ }^{21}\right)$ of a CC algebra is CC. Hence if $M$ is a regular maximal ideal (which is closed by Corollary 1.6.1) the $B$-quotient algebra $A / M$ is $\mathrm{CC}$, and has an identity. Clearly, the unit sphere in $A / M$ is compact and by F. Riesz' theorem about LC linear spaces, $A / M$ is finite-dimensional. Putting $K$ for a regular maximal left ideal which contains $M$ (by Theorem 1.2 suç an ideal exists), it follows that $A-K$ is finite-dimensional. It is easy to show, from the fact that $M$ is maximal, that $[x \mid x A \subset K]=M$. Reference to Theorem 1.8 completes the proof that $M$ is the kernel of the extension of a bounded, continuous, irreducible representation of $G$ by finite-dimensional matrices.

Now suppose that $\phi$ is such a representation of $G$. By Burnside's theorem, the algebra of matrices generated by the range $R$ of $\phi$ is a full matrix algebra. We now show that, on the other hand, the image of $A$ under the extension $\Phi$ of $\phi$ is dense in $R$. It will then follow, clearly, that $\Phi(A)$ is the same full matrix algebra. Let $g$ be an arbitrary continuous complex-valued function on $G$, let $V$ be an arbitrary neighborhood of the identity in $G$, and put $h_{V}$ for the characteristic function of $V$, divided by the measure of $V$. It is easy to verify that $\lim _{V} \int_{G} g(a) h_{V}\left(a b^{-1}\right) d a=g(b)$, the limit being taken in the MooreSmith sense, with set inclusion reversed as the partial ordering on the system of neighborhoods. Plainly, $\Phi\left(h_{V}^{b}\right)=\int_{G} \phi(a) h_{V}\left(a b^{-1}\right) d a$, and therefore lim $\Phi\left(h_{V}^{b}\right)$ $=\phi(b)$, completing the proof that $\Phi(A)$ is dense in the range of $\phi$.

Since a full matrix algebra has an identity and is simple, the kernel of $\Phi$ is regular and maximal. It is evident that the kernels of the extensions of two representations of the type described in the theorem are the same in case the representations are similar. Conversely, if the kernels of the extensions of two representations are the same, the images of $A$ under the extensions are clearly isomorphic. Since every automorphism of a full matrix algebra is inner, the representations are similar.

In the case of an abelian group, it follows easily from the Gelfand-Mazur theorem [10] (that a simple commutative Banach algebra is isomorphic as a topological algebra with the complex numbers) that if $M$ is a regular maximal

${ }^{(20)}$ In the case of a LC abelian group such representations must, of course, be continuous characters.

(21) The $B$-quotient algebra $B / I$ of a $B$-algebra $B$ with respect to the closed ideal $I$ is the $B$-quotient space $B-I$ with multiplication defined by $(a+I)(b+I)=a b+I$. It is easy to verify that a $B$-quotient algebra is a $B$-algebra (cf. footnote 18 ). 
ideal, the representation of $G$ given by Theorem 1.8 (with $K=M$ ) is onedimensional. Being bounded and continuous, the representation is a continuous character. It is clear, on the other hand, that the kernel of such a representation is regular and maximal. The argument used above in the case of a compact group to show that inequivalent representations correspond to different ideals also applies to the case of an abelian group.

6. Some comments on the foregoing theory. The purpose of this section is to present two theorems which, it is hoped, clarify somewhat the limits and significance of some of the preceding methods. To begin with, the notion of adverse is required in order to deal effectively with principal ideals in an algebra without identity; in connection with this observation the following theorem is of interest.

THEOREM 1.10. The group algebra of a LC group has an identity if and only if the group is discrete.

Putting $G$ for the group, $A$ for the group algebra, and $i$ for the group identity, it is easy to verify that if $G$ is discrete, the element $f(\cdot)$, defined by $f(i)=1, f(x)=0$ when $x \neq i$, is an identity in $A$. Now suppose that $A$ has an identity $f(\cdot)$; then:

$$
\int_{G} g\left(y x^{-1}\right) f(y) d y^{-1}=g(x)
$$

almost everywhere, if $g(\cdot) \in A$. In case $g(\cdot)$ is a continuous function that vanishes outside a compact set, it is clear that both sides of the preceding equation represent continuous functions, and therefore the equation holds for all values of $x$. Setting $x=i$, we get $\int_{G} g(y) f(y) d y^{-1}=g(i)$, for such $g(\cdot)$. If we put $\phi(E)=\int f(y) d y^{-1}$, where $E$ is a general measurable set with compact closure, and define $\mu(E)=1$ if $i \in E, \mu(E)=0$ otherwise, then, evidently,

$$
\int_{G} g(y) d \phi\left(E_{y}\right)=\int_{G} g(y) d \mu\left(E_{y}\right),
$$

where $g(\cdot)$ is an arbitrary continuous function vanishing outside of a compact set. By a uniqueness theorem for measures on $L C$ spaces, $\phi(\cdot)=\mu(\cdot)$. Since $\phi(\cdot)$ is absolutely continuous with respect to Haar measure, this equality implies that $\{i\}$ has positive Haar measure. It follows that every infinite set has infinite Haar measure. Therefore a compact subset is finite; and from the fact that $G$ is LC it is easy to conclude that a set consisting of a single point is open.

It is plain that the methods of proof of Theorem 1.9 apply to any group algebra that is either $\mathrm{CC}$ or abelian. However, this observation cannot be utilized to extend the scope of that theorem, as the following theorem shows.

THEOREM 1.11. The group algebra of a LC group is (CC, abelian) if and only if the group is (compact, abelian). 
Let $G$ be the group and $A$ the group algebra. Assuming that $A$ is CC, let $f$ be a general element of $A$ and put $T$ for its right regular representation. By a theorem of Dunford and Pettis about BDH integrals [9, Theorem 3.1.10], the fact that $T$ is CC implies the existence of a null set $N$ such that $U=\left[f_{y} \mid y \in G-N\right]$ is totally bounded, where $\left(f_{y}\right)(x)=(f)\left(y^{-1} x\right)$. Since $G-N$ is dense in $G$ and the mapping $y \rightarrow f_{y}$ is continuous on $G$ to $A$, the closure of $U$ contains $V=\left[f_{y} \mid y \in G\right]$; hence $V$ is totally bounded. Therefore, there exists for an arbitrary positive number $e$ a finite set of elements of $G,\left(a_{1}, \cdots, a_{n}\right)$, such that $U_{i}\left[a \mid\left\|f_{a}-f_{a_{i}}\right\|<e\right]=G$. Now let $P$ be a neighborhood of the identity with finite measure, and such that $Q Q^{-1} \subset P$. Taking $f$ to be the characteristic function of $Q$ and $e$ to be less than $m(Q)$, where $m(\cdot)$ is Haar measure, it follows that $\left\|f_{a}-f_{a_{i}}\right\|=m\left(a Q \circ a_{i} Q\right)$, where $\circ$ designates the symmetric difference. Hence $m\left(a Q \circ a_{i} Q\right)<m(Q)=m(a Q)$; and clearly, $a Q \cap a_{i} Q \neq 0$, $a \in a_{i} P, G \subset \mathrm{U}_{i} a_{i} P$. Thus $G$ is totally bounded, and since it is LC, $G$ is compact. On the other hand, it follows easily, as noted previously, that if $G$ is compact then $A$ is CC.

Now suppose that $A$ is abelian. Defining $\rho(\cdot)$ by the relation $d y^{-1}=\rho(y) d y$ and writing out the equation $f g=g f$ using the definition of convolution, it results that:

$$
\int_{\theta} f(y)\left[g\left(y^{-1} x\right)-\rho(y) g\left(x y^{-1}\right)\right] d y=0,
$$

for all $x$ except a null set. From the arbitrary character of $f(\cdot)$, it follows that

$$
g\left(y^{-1} x\right)=\rho(y) g\left(x y^{-1}\right),
$$

for all $x$ except a null set, for almost all values of $y$. If $g(\cdot)$ is continuous, the equation holds for all values of $x$ and $y$, the complement of a null set being dense in $G$. Setting $x$ equal to the identity shows that $\rho(y)^{\prime}=1$. It is easy to conclude from the general character of $g(\cdot)$ (any continuous integrable function) that $y^{-1} x=x y^{-1}$. On the other hand, it is obvious that if $G$ is abelian, then so is $A$.

\section{Part II. Spectral Resolution of IDEALS IN GROUP ALGEBRAS}

1. General algebras. We derive an approximate decomposition for an ideal in an algebra into regular maximal ideals, modulo the radical of the algebra, which specializes to a kind of spectral resolution in the case of the group algebra of the real line or the algebra generated by a symmetric matrix. In later sections of this part this decomposition, which we call the spectral resolution, is applied to ideals in the group algebra of a group that is either LC abelian, compact, or discrete.

DEFINITION 2.1. Let $\Gamma$ be the family of all regular maximal ideals of an algebra $A$. Let $\Delta$ be a general subset of $\Gamma$ and $I$ an ideal in $A$. The kernel of $\Delta$ is the intersection of all ideals in $\Delta$, except that it is $A$ when $\Delta$ is void. 
The hull of $I$ is the set of regular maximal ideals containing it. The spectrum of $I$ is the complement in $\Gamma$ of its hull. The hull or spectrum of a set of elements is that of the ideal generated by them. $\Delta$ is said to be closed if it equals the hull of its kernel. A neighborhood of infinity is the complement of a set which is such that there exists an element of $A$ which has an inverse modulo every element of the set. With the preceding family of closed sets, $\Gamma$ is called the spectrum of $A$.

THEOREM 2.1. The spectrum of an algebra is a $T_{1}$ topological space.

The notation of Definition 2.1 is utilized. It is clear that the null set is closed and that the intersection of any family of closed sets is closed. To show that the union of a finite family of closed sets is closed an indirect proof is used. Let $\Delta_{i}$ be closed $(i=1, \cdots, n)$ and assume that $\Delta=U_{i} \Delta_{i}$ is not closed. Putting $D$ for the kernel of $\Delta$ and $D_{i}$ for the kernel of $\Delta_{i}$, it follows that there exists an element of $\Gamma, M$, which contains $D$ and is not in $\Delta$. Plainly, $M$ is not in $\Delta_{i}$, and therefore the ideal generated by $M$ and $D_{i}$ is $A$. It follows that if $e$ is an identity modulo $M$, there exist $x_{i} \in D_{i}$ and $y_{i} \in M$ such that $x_{i}+y_{i}=e(i=1, \cdots, n)$. Multiplying together these equations, it is easily seen that $x_{1} x_{2} \cdots x_{n} \equiv e$ modulo $M$. On the other hand, since $D \subset M, x_{1} x_{2} \cdots x_{n} \in M$, contradicting the preceding congruence. That the space is $T_{1}$ follows from the fact that an inclusion relation between maximal ideals can exist only if they are the same.

Now the spectral resolution for ideals is proved.

THEOREM 2.2. An ideal in an algebra contains, modulo the radical of the algebra, the kernel of an open set which contains both the hull of the ideal and a neighborhood of infinity.

Let $I$ be an ideal in the algebra $A, \Omega$ its hull, $\Lambda$ an open set in the spectrum $\Gamma$ of $A$ which contains both $\Omega$ and a neighborhood of infinity, and put $\Delta=\Gamma-\Lambda, D$ for the kernel of $\Delta$, and $f(\cdot)$ for the homomorphism on $A$ to $A / D$. Clearly there exists an element $e$ of $A$ that is an identity modulo every element of $\Delta$. It follows that $e$ is an identity modulo $D$, and that $f(e)$ is an identity in $A / D$. By Theorem 1.2, either $f(I)$ is contained in a maximal ideal in $A / D$, or $f(I)=A / D$. It is easy to verify that a maximal ideal in $A / D$ has the form $M / D$ with $M \in \Delta$; and $f(I) \subset M / D$ implies $I \subset M$, which is impossible. Hence $f(I)=A / D$, and it follows that $I$ contains an element $e^{\prime}$ which is an identity modulo $D$. Now if $x$ is in the kernel of $\Lambda$, it is trivial to verify that $x e^{\prime} \equiv x$ modulo $M$ for every $M \in \Gamma$, or $x e^{\prime} \equiv x$ modulo the radical. This concludes the proof, it being obvious that $x e^{\prime} \in I$.

Corollary 2.2.1. If $I$ is a closed ideal in a topological algebra $A$ with the property that elements whose hulls contain neighborhoods of infinity are dense and such that $x$ is in the closure of $x A$ for every $x \in A$, then I contains, modulo the radical, the kernel of an open set containing the hull of $I$. 
Let $\Lambda$ be an open set containing the hull of $I$, and put $L$ for the kernel of $\Lambda$. It is plain from Theorem 2.2 that if $y \in L$, and if the hull of $x$ contains a neighborhood of infinity, then $y x \in I$. It follows that $y u \in I$ for every $u \in A$, because $I$ is closed and elements whose hulls contain neighborhoods of infinity are dense, and hence $y \in I$, since $y \in(y A)^{c}$.

The following theorem is useful for the later discussion of the group algebra of a discrete group.

THEOREM 2.3. The spectrum of an algebra which has an identity modulo its radical is compact.

Suppose that $e$ is an identity modulo the radical of the algebra $A$ and that $\bigcap_{s \in S} \Delta_{s}=0$, where $\left\{\Delta_{s}, s \in S\right\}$ is a family of closed subsets of $\Gamma$. Of course, the compactness of $\Gamma$ is equivalent to the existence of a finite subfamily whose intersection is void. Put $D_{s}$ for the kernel of $\Delta_{s}$ and $D$ for the ideal union of the $D_{s}, s \in S$. Plainly, $e$ is an identity modulo $D$. Hence, by Theorem 1.2 , either $D=A$ or $D$ is contained in a regular maximal ideal $M$. It is easy to verify that the latter condition implies $M \in \bigcap_{s} \in S \Delta_{s}$, which is impossible. Hence $D=A$, implying that the $e$ has the form $\sum_{t \in T} x_{t}$, where $x_{t} \in D_{t}$ and $T$ is finite. It follows readily (noting that $D_{s}$ contains the radical) that $\bigcap_{t} \in T \Delta_{t}=0$, concluding the proof.

2. The spectrum of a group algebra. The application to ideals in a particular algebra of the preceding spectral resolution is naturally strengthened by determination in more explicit fashion of the topology on the spectrum. The present section is concerned partly with this topology in the case of the group algebra of a group that is either LC abelian, compact, or discrete, and partly with the verification of the conditions for the resolution of closed ideals in such group algebras. Theorem 2.4 suggests that the spectrum of a group algebra may be dual to the group in a fashion somewhat analogous to that in the case of the duality between a LC abelian group and its character group.

THEOREM 2.4. The spectrum of the group algebra of the locally compact group $G$ is:

(a) homeomorphic with the character group, via the mapping defined by Theorem 1.10, in case $G$ is abelian (22);

(b) discrete, in case $G$ is compact;

(c) compact, in case $G$ is discrete.

First let $G$ be abelian, put $G^{*}$ for its continuous character group, and $\phi(\cdot)$ for the mapping on $G^{*}$ to the spectrum $\Gamma$ of the group algebra defined by Theorem 1.10. It is plain from Theorem 1.9 that $\phi(\cdot)$ is biuniform. To show

(22) We regard the group $G^{*}$ as topological, the topology being that of Pontryagin, specifically: a general neighborhood of the point $x_{0}^{*}$ of $G^{*}$ is $\left[x^{*}\left|\sup _{x \in c}\right| x^{*} x-x_{0}^{*} x \mid<e\right]$ where $C$ is a general compact subset of $G$ and $e$ is a general positive number. 
that $\phi(\cdot)$ is continuous an indirect argument is used: assume that $y^{*} \in \bar{S}^{*}$ and $\phi\left(y^{*}\right) \notin\left(\phi\left(S^{*}\right)\right)^{c}$. Then for some $f(\cdot) \in A$, the group algebra, $\int_{G} y^{*} x f(x) d x \neq 0$ and $\int_{G} x^{*} x f(x) d x=0$ for $x^{*} \in S^{*}$. Defining

$$
e=4^{-1}\left|\int_{G} y^{*} x f(x) d x\right|, \quad e^{\prime}=e\left(\int_{G}|f(x)| d x\right)^{-1},
$$

let $C$ be a compact subset of $G$ such that $\int_{G_{-} C}|f(x)| d x<e$ and put $N^{*}=\left[x^{*}|| y^{*} x-x^{*} x \mid<e^{\prime}\right.$ for all $\left.x \in C\right]$. If we express $\int_{G} y^{*} x f(x) d x$ as

$$
\int_{C}\left(y^{*} x-x^{*} x\right) f(x) d x+\int_{G-C}\left(y^{*} x-x^{*} x\right) f(x) d x+\int_{G} x^{*} x f(x) d x,
$$

and now choose $x^{*} \in N^{*} \cap S^{*}$, a contradiction results:

$$
\left|\int_{G} y^{*} x f(x) d x\right|<3 e .
$$

To show that $\phi^{-1}(\cdot)$ is continuous, an indirect argument is again used: assume that $M \in\left(\phi\left(S^{*}\right)\right)^{c}, \phi^{-1}(M) \in \bar{S}^{*}(M \in \Gamma)$. Let $U^{*}$ be a neighborhood of the identity in $G^{*}$ such that $\bar{U}^{*}$ is compact and $S^{*} \cap y^{*} U^{*} U^{*-1}=0$, where $y^{*}=\phi^{-1}(M)$. Let $H$ be the characteristic function of $U^{*}$ and put $F(\cdot)$ for the convolution of $H$ with $H^{*}$ with respect to Haar measure on $G^{*}$. By the theory of the Plancherel-Weil transform, $F\left(x^{*}\right)=\int_{G} x^{*} x f(x) d x$ for some $f(\cdot) \in A$. Setting $g(x)=\left(y^{*} x\right)^{c} f(x)$, it is clear that $\int_{G} y^{*} x g(x) d x \neq 0, \int_{G} x^{*} x y(x) d x$ $=0$ for $x^{*} \in S^{*}$, and $g(\cdot) \in A$, contradicting the assumption that $M \in\left(\phi\left(S^{*}\right)\right)^{c}$.

Now let $G$ be compact. To show that $\Gamma$ is discrete is plainly equivalent to showing that for a general $M \in \Gamma, M D \cap_{I \in \Gamma, I \neq M} I$. By Theorem 1.9, $M$ is the kernel of the extension of a finite-dimensional bounded continuous irreducible representation of $G$. Putting $f(\cdot)$ for any coordinate of a unitary representation equivalent to this representation, it is plain that $(f(\cdot))^{\circ} \in A$, and it follows readily from the orthogonality relations $\left({ }^{23}\right)$ that $(f(\cdot))^{c} \notin M$, $(f(\cdot))^{\circ} \in \bigcap_{I \in \Gamma_{1} I \neq M} I$.

It follows immediately from Theorems 1.10 and 2.3 that $\Gamma$ is compact in case $G$ is discrete.

THEOREM 2.5. The complement of a compact set, in the spectrum of the group algebra of a group which is either LC abelian, compact, or discrete, contains a neighborhood of infinity.

In case the group $G$ is $L C$ abelian it is plainly sufficient to show that for a general compact subset $C^{*}$ of the character group, there exists an element of the group algebra $A$ whose Fourier transform is equal to unity at each point of $C^{*}$. Let $U^{*}$ be a conditionally compact open set containing $C^{*}$, put

(2) See, for example, [35, p. 73]. 
$V^{*}=U^{*} U^{*-1}$, and let $f(\cdot)$ and $g(\cdot)$ be the characteristic functions of $U^{*}$ and $V^{*}$ respectively. Setting $h(\cdot)=m^{-1} f g$, where $m$ is the Haar measure of $U^{*}$ (and $f g$ is the convolution of $f$ and $g$ with respect to Haar measure on $G^{*}$ ), it is easy to verify that $h\left(x^{*}\right)=1$ for $x^{*} \in C^{*}$. Plainly $f$ and $g$ are PlancherelWeil transforms of elements of $L_{2}(G), \phi(\cdot)$ and $\gamma(\cdot)$, and it follows that $h(\cdot)$ is the Fourier transform of the function $m^{-1} \phi(x) \cdot \gamma(x)$; and it is obvious that this function is an element of $A$.

In the case of a compact group it plainly suffices to show that if $\left[m_{i}(\cdot) \mid i=1, \cdots, n\right]$ is a general finite set of continuous irreducible representations, then there exists an element of $A, f(\cdot)$, such that $\int_{G f} f(x) m_{i}(x) d x$ is the identity matrix $(i=1, \cdots, n)$. It follows easily from the orthogonality relations that the complex conjugate of the sum of the diagonal coordinates of the $n$ representations is such an element.

For discrete groups the conclusion follows immediately from Theorems 1.10 and 2.3, and Definition 2.1.

THEOREM 2.6. Let $A$ be the group algebra of a group that is either LC abelian, compact, or discrete. Then the class of elements of $A$ whose hulls contain neighborhoods of infinity is dense.

First let the group $G$ be LC abelian, let $f(\cdot)$ be a general element of $A$, and let $f_{i}(x)(i=1,2)$ be elements of $L_{2}(G)$ such that $f(x)=f_{1}(x) f_{2}(x)$-clearly such elements exist. Taking inverse Plancherel-Weil and Fourier transforms, $\phi=\phi_{1} \phi_{2}$, where $\phi_{i}$ is the transform of $f_{i}$ (and $\phi_{1} \phi_{2}$ is the convolution of $\phi_{1}$ and $\phi_{2}$ on $G^{*}$ ). Plainly, for a general positive number $e$ there exist elements of $L_{2}\left(G^{*}\right), \gamma_{1, e}$ and $\gamma_{2, e}$, which vanish outside compact sets and are such that $\left\|\gamma_{i, e}-\phi_{i, e}\right\|_{2}<e$, where $\|\cdot\|_{2}$ is the $L_{2}$ norm. It is easy to verify that $\gamma_{1, e} \gamma_{2, e}$ is the Fourier transform of an element $g_{\theta}$ of $A$, and that $\left\|f-g_{\theta}\right\| \rightarrow 0$ as $e \rightarrow 0$. By Theorem 2.5 this completes the proof for the case of an abelian group.

In the case of a compact group it is plainly sufficient, by Theorem 2.5, to show that the elements of $A$ whose spectrum is a finite set is dense. An indirect proof is utilized: assume that the span of the preceding class of elements is a proper subspace of $A$. By the Hahn-Banach theorem and Riesz' representation theorem for continuous linear functionals on $A$, there exists an essentially bounded measurable function $g(\cdot)$ on $G$ which is not zero and such that $\int_{G} g(x) f(x) d x=0$ for every $f(\cdot) \in A$ of finite spectrum. Since the measure of $G$ is finite, $g(\cdot) \in A$. On the other hand, it is clear from the orthogonality relations that the complex conjugate of a coordinate of a continuous irreducible representation of $G$ by matrices has a finite spectrum, which by Theorems 1.7 and 1.9 implies that $g(\cdot)=0$, a contradiction.

The proof is trivial for the case of a discrete group.

COROLlaRy 2.6.1. The group algebra of a group which is either LC abelian, compact, or discrete satisfies the conditions of Corollary 2.2.1. 
3. The group algebra of the reals. Theorems 1.7 and 2.4 and Corollary 2.6.1 show that a closed ideal in the group algebra of a compact group equals the kernel of its hull. In the present section it is shown that a similar, though weaker, theorem holds for the group algebra of the real line-more specifically the same conclusion is valid provided the hull has a certain topological character $\left({ }^{24}\right)$.

THEOREM 2.7. Let I be a closed ideal in the group algebra of the additive group of the real numbers. Let $\Delta$ be the hull of $I$ and put $D$ for the closed set of real numbers homeomorphic with $\Delta$ by Theorem 1.9. Suppose that $D=P+Q$, where $P$ is a closed set without limit points and $Q$ is a union of (possibly semiinfinite) open intervals. Then I equals the hull of its kernel.

Two main tools are employed in the proof: a family of special functions utilized considerably by Wiener in his harmonic analysis investigations, and an inequality of Carlson and Beurling. The special functions are a kind of approximate idempotents (the group algebra contains, of course, no actual idempotents except zero) and the family of these functions plays a part analogous to that of the minimal idempotents in the theory of algebras of finite dimension. The inequality is needed because it is algebraically more effective to consider the Fourier transform of an element of the group algebra $A$ (rather than the element itself) but analytically difficult because the norm of an element is not expressible in a simple fashion in terms of the Fourier transform of the element. The Carlson-Beurling inequality bounds the norm by a functional expressible in a highly direct way in terms of the Fourier transform. The basic lemma is first proved for elements on which this functional is finite; the fact that such elements are dense in $A$ is then used to conclude the proof.

LEMMA 2.7.1. If we define

$$
\lambda(x, s, p)=\left(\frac{8}{\pi}\right)^{1 / 2} e^{-i p x} \frac{1-\cos x s}{x^{2} s} \cos \frac{x s}{2},
$$

then: (a) $\lambda(\cdot, s, p$ ) $\in A$ if $s$ is positive and $p$ is real, (b) $\lambda(\cdot, s, p) f \rightarrow 0$ as $s \rightarrow 0$, if $f$ is an element of $A$ whose Fourier transform vanishes at $p$, (c) the Fourier transform of $\lambda(\cdot, s, p)$ is:

$$
\Lambda(t, s, p)= \begin{cases}1 & \text { if }|t-p|<s, \\ 1-s^{-1}|t-p| & \text { if } s \leqq|t-p|<2 s, \\ 0 & \text { if }|t-p| \geqq 2 s .\end{cases}
$$

It is easy to verify that it is sufficient to consider the case $p=0$. It is

(24) A similar theorem has been proved in [8], according to the review of this paper in Mathematical Reviews. 
trivial that $\lambda(\cdot, s, 0), \lambda_{s}$ say, is an element of $A$ if $s$ is positive and that $\left\|\lambda_{s}\right\|$ is bounded as $s \rightarrow 0$. It is well known that $\lambda(\cdot, s, 0)$ is the Fourier transform of $\lambda_{s}$ (see, for example, Wiener [37, pp. 49-50]). Therefore, to conclude the proof of the lemma it suffices to show that $\lambda_{s} f \rightarrow 0$ as $s \rightarrow 0$ for all $f$ in a set which is dense in the class of elements of $A$ whose Fourier transform vanishes at zero. Now the set $B$ of all functions which are finite linear combinations of characteristic functions of bounded intervals and whose Fourier transform vanishes at zero is easily seen to be such a set.

The Carlson-Beurling inequality $[7,4]$ states that for $g(\cdot) \in A$ :

$$
\int_{-\infty}^{\infty}|g(x)| d x \leqq\left(\int_{-\infty}^{\infty}|G(t)|^{2} d t\right)^{1 / 4}\left(\int_{-\infty}^{\infty}\left|G^{\prime}(t)\right|^{2} d t\right)^{1 / 4},
$$

where $G(\cdot)$ is the Fourier transform of $g(\cdot)$, provided that $G(\cdot)$ is absolutely continuous. Setting $g(\cdot)=\lambda_{s} f$, where $f$ is a general element of $B$, it follows readily that $\lambda_{s} f \rightarrow 0$ as $s \rightarrow 0$.

Resuming the proof of the theorem, suppose first that $f$ is an element of $A$ whose Fourier transform, $F$, vanishes on $D$, and also on a neighborhood of infinity $N$. Plainly, there exists a finite set of points in $D,\left(p_{1}, \cdots, p_{k}\right)$, such that the (set) union of $U_{s}\left(p_{i}\right)(i=1, \cdots, k), N$, and $Q$ is an open set $V$ containing the hull of $f$, where $U_{s}(p)=(p-s, p+s)$. Clearly, $\prod_{i=1}^{k}\left(1-\Lambda\left(\cdot, s, p_{i}\right)\right) F$ (pointwise product) is the Fourier transform of an element $f_{s}$ of the kernel of $V$. By Theorem 2.2, $f_{s} \in I$; and if we utilize Lemma 2.7.1, it is easy to show that $f \in I$. Now it follows from Theorem 2.6 that the conclusion is valid without the hypothesis that $F$ vanishes on a neighborhood of infinity, completing the proof.

4. Some theorems on the spectrum of a general algebra. The theorems presented in this section may be useful in connection with further investigations of the ideal theory of group algebras, and are of independent interest.

THEOREM 2.8. Let $\bar{A}$ be the algebra obtained from the algebra $A$ by adjoining an identity as follows: $\bar{A}$ consists of all ordered pairs $(x, \alpha)$ with $x \in A$ and $\alpha \in F, F$ being the field of $A$, and algebraic operations defined by

$$
\begin{gathered}
\alpha(y, \beta)=(\alpha y, \alpha \beta), \quad(x, \alpha)+(y, \beta)=(x+y, \alpha+\beta), \\
(x, \alpha)(y, \beta)=(x y+\alpha y+\beta x, \alpha \beta) ;
\end{gathered}
$$

here $x$ and $y$ are general elements of $A$ and $\alpha$ and $\beta$ general elements of $F$. Then the spectrum of $A$ is homeomorphic with the relative space obtained from the (compact) spectrum of $\bar{A}$ by deleting the (regular) maximal ideal $A^{\prime}=[(x, 0) \mid x \in A]$, and $A$ is isomorphic with $A^{\prime}$.

It is easy to verify that $\bar{A}$ is an algebra with $(0,1)$ as identity and that the mapping $x \rightarrow(x, 0)$ is an isomorphism on $A$ to $A^{\prime}$. Clearly $A^{\prime}$ is an ideal in $\bar{A}$, and $\bar{A} / A^{\prime} \sim F$; hence $A^{\prime}$ is maximal. To avoid circumlocution, $A^{\prime}$ will 
now be identified with $A$. For a point $M$ in the spectrum $F$ of $A$, put $\bar{M}=f(M)=\left[(x, \alpha) \mid x+\alpha e_{M} \in M\right]$, where $e_{M}$ is an identity modulo $M$. Plainly $\bar{M}$ is an ideal in $\bar{A}$, and by the isomorphism law for ideals:

$$
(\bar{M}+A) / \bar{M} \sim A /(\bar{M} \cap A) .
$$

Now it is easy to show that $\bar{M} \cap A=M$ and $\bar{M}+A=\bar{A}$. It follows that $\bar{M}$ is maximal in $\bar{A}$.

To show that $f(\cdot)$ is uniform, suppose $f\left(M_{1}\right)=f\left(M_{2}\right)$, where $M_{1}$ and $M_{2}$ are in $\Gamma$. Then $x+\alpha e_{1} \in M_{1}$ is equivalent to $x+\alpha e_{2} \in M_{2}$, where $e_{i}$ is an identity modulo $M_{i}$; in particular, $x \in M_{1}$ is equivalent to $x \in M_{2}$, or $M_{1}=M_{2}$. On the other hand, if $P$ is a regular maximal ideal in $\bar{A}$ different from $A$, it follows from the isomorphism law for ideals that $P \cap A$ is a regular maximal ideal $M$ in $A$. Putting $e$ for an identity modulo $M,(x, \alpha) \in P$ implies $(x, \alpha)(e, 0) \in P$, or $(x e+\alpha e, 0) \in P$, which shows that $x+\alpha e \in M$. It follows that $P \subset f(M)$, and $P=f(M)$ since both ideals are maximal, completing the proof that $f(\cdot)$ is biuniform.

To prove the continuity of $f(\cdot)$, let $\Delta$ be a general subset of $\Gamma$ and put $D$ and $\bar{D}$ for the kernels of $\Delta$ and $f(\Delta)$ respectively. It is necessary to show that if $M_{0} \in \Gamma$ and $M_{0} \supset D$, then $\bar{M}_{0}^{\prime} \supset \bar{D}^{\prime}$. Let $(x, \alpha)$ be a general element of $\bar{D}$. Then $x+\alpha e_{M} \in M$ for every $M \in \Delta$, where $e_{M}$ is an identity modulo $M$. Multiplying by an identity $e$ modulo $M_{0}, x e+\alpha e \in M$ for every $M \in \Delta$, which shows that $x e+\alpha e \in M_{0}$, and therefore $(x, \alpha) \in \bar{M}_{0}$. To prove that $f^{-1}(\cdot)$ is continuous it is necessary to show that $\bar{M}_{0} \supset \bar{D}$ implies $M_{0} \supset D$, which follows by intersecting both $\bar{M}_{0}$ and $\bar{D}$ with $A$, completing the proof.

It follows that only a quite special $T_{1}$-space-a space which is close to being LC-can possibly be the spectrum of an algebra $\left({ }^{25}\right)$ :

COROLlaRy 2.8.1. The spectrum of an algebra is homeomorphic with the relative space obtained by deleting a point from a compact $T_{1}$-space.

It has been shown above that the complement of a compact set in the spectrum of a group algebra of a group which is either LC abelian or compact contains a neighborhood of infinity. It is natural to ask whether the converse is true, that is, whether the complement of a neighborhood of infinity is contained in a compact set. It follows from the next theorem that this is actually the case. Hence, if infinity is added to the spectrum of such an algebra as an ideal point, the spectrum is compactified in the manner usual in topology, and our term "neighborhood of infinity" is justified.

THEOREM 2.9. $A$ closed set $\Delta$ in the spectrum of an algebra $A$ is homeomorphic with the spectrum of $A / D$, where $D$ is the kernel of $\Delta$.

(26) The spectrum is not necessarily a Hausdorff space, an example being the spectrum of the algebra of all polynomials over the complex field. It is easy to see that the spectrum of this algebra is in one-to-one correspondence with the complex plane, and that a general non-null open set consists of the complement of a general finite set of points. 
Let $f(\cdot)$ be the homomorphism on $A$ to $A / D$, and for $M \in \Delta$ define $M_{D}=f(M)$. By the isomorphism laws for ideals, $A / M \sim(A / D) / M_{D}$, showing that $M_{D}$ is in the spectrum $\Gamma_{D}$ of $A / D$. On the other hand, if $I_{D} \in \Gamma_{D}$, then clearly $f^{-1}\left(I_{D}\right) \in \Delta$. Thus $f(\cdot)$ is biuniform on $\Delta$ to $\Gamma_{D}$.

Now if $\Lambda$ is an arbitrary subset of $\Delta$, it is clear that $M_{0} \supset \cap_{M \in \Delta} M, M_{0} \in \Delta$ imply $f\left(M_{0}\right) \supset \bigcap_{M \in A} f(M)$; hence $f(\cdot)$ is continuous on $\Delta$ to $\Gamma_{D}$. If $\mathbf{P}_{D} \in \Gamma_{D}$ and $\mathrm{P}_{D} \supset \cap_{M \in \Delta} M_{D}$, then clearly $\mathrm{P} \supset \cap_{M_{\Delta}} M$; hence $f(\cdot)$ is bicontinuous, and the proof is complete.

COROLlaRY 2.9.1. The complement of a neighborhood of infinity is compact.

Putting $\Lambda$ for a neighborhood of infinity in the spectrum of the algebra $A$ and $D$ for the kernel of the complement $\Delta$ of $\Lambda$, it is easy to verify that $\Delta$ is closed. Plainly $A / D$ has an identity, and therefore by Theorem 2.3 its spectrum is compact. It follows from the theorem that $\Delta$ is compact.

\section{PART III. ApPlications to haRmonic anAlysis ON GROUPS}

1. Tauberian theorems. Straightforward application of the spectral resolution of ideals in the group algebra of the additive group of the real numbers results in a very considerable generalization of Tauberian theorems due to Wiener and to Pitt. In addition, the proofs of the previously known theorems are simplified to an extent which can hardly be overestimated. With regard to the historical development of this method, some aspects were implicit in Wiener's work. On the other hand, a crucial aspect-the utilization of properties of maximal ideals in algebras-originated in the work of Gelfand and his associates. Specifically, the fact that a proper ideal in an algebra with an identity is contained in a maximal ideal (which is due to Krull) was basic in Gelfand's Banach algebra investigations, and was applied by him to prove the main lemma in Wiener's proof of his general Tauberian theorem. In the present section a theorem of Tauberian type is proved which includes Wiener's general Tauberian theorem and a related theorem due to Pitt as very special cases, and it is clear from the proof that a wide class of Tauberian theorems consists essentially of theorems about the ideal theory of the group algebra of the reals.

THEOREM 3.1. Let $B$ be a dense linear subspace of the group algebra $A$ of the additive group of the real numbers and a Banach space with respect to a norm $\|\cdot\|$ (not necessarily the same as the norm on $A$ ). Assume that $B$ satisfies the following three conditions:

(a) $f \in B$ implies that $f_{a} \in B$, where $\left(f_{a}\right)(x)=(f)(x-a)$, for every real a, and $\left\|f_{a}\right\|=\|f\|$.

(b) The mapping $a \rightarrow f_{a}$ on the real numbers to $B$ is continuous for $f \in B$.

(c) If $\left\|f_{n}\right\| \rightarrow 0$, then $\int_{-\infty}^{\infty}\left|\left(f_{n}\right)(x)\right| d x \rightarrow 0$.

Let $\Omega$ be the unit sphere in the conjugate space of $B$ and define 


$$
\begin{aligned}
G(w, f) & =\limsup _{a \rightarrow \infty}\left|w f_{a}\right|, \\
\phi\left(G, f_{1}, f_{2}\right) & =\underset{w \in \Omega, G\left(w, f_{1}\right) \leqq G}{\text { L.U.B. }} G\left(w, f_{2}\right) .
\end{aligned}
$$

Let $f_{1}$ and $f_{2}$ be elements of $B$,put $Z_{i}$ for the set of real zeros of the L-Fourier transform of $f_{i}$, and assume either (i) $Z_{1}$ is in the interior of $Z_{2}$, or (ii) $Z_{1} \subset Z_{2}$, and $Z_{1}=P+Q$, where $P$ is a closed set without limit points, $Q$ is a union of open intervals, and $P$ and $Q$ are disjoint.

Then: $\lim _{G \rightarrow 0} \phi\left(G, f_{1}, f_{2}\right)=0$.

It is plain that if $f \in A$ and $g \in B$, the BDH integral

$$
\int_{-\infty}^{\infty} g_{x} f(x) d x
$$

exists, and it is easy to verify that it equals $f g$. Hence $B$ is an ideal in $A$. Now set $I=\left[f \mid f \in B, \lim _{G \rightarrow 0} \phi\left(G, f_{1}, f_{2}\right)=0\right]$. It is easy to show that $\phi\left(G, f_{1}, f+g\right) \leqq \phi\left(G, f_{1}, f\right)+\|g\|$, from which it follows that $I$ is closed in $B$. The following equations and inequalities show that $I$ is an ideal in $A$ :

$$
\begin{aligned}
G(w, f g) & =\limsup _{a \rightarrow \infty}\left|w \int_{-\infty}^{\infty} f_{a+b} g(b) d b\right| \\
& =\limsup _{a \rightarrow \infty}\left|\int_{-\infty}^{\infty} w f_{a+b} g(b) d b\right| \\
& \leqq \int_{-\infty}^{\infty} \lim \sup \left|w f_{a+b} g(b)\right| d b \\
& \leqq G(w, f) \int_{-\infty}^{\infty}|g(b)| d b .
\end{aligned}
$$

Now observe that $\bar{I} \cap B=I$, where $\bar{I}$ is the closure of $I$ in $A$. Because if $x \in \bar{I} \cap B$, let $\left\{x_{n}\right\}$ be a sequence of elements of $A$ whose $A$-limit is $x$. It follows from the theory of the BDH integral that $\left\|x_{n} y-x y\right\| \rightarrow 0$ for $y \in A$, showing that $x y \in I$ for $y \in A$. It is clear from the continuity of $f_{a}$ as a function of $a$ that if $e$ is a positive number then there exists $y_{e} \in A$ such that $\left\|x-x y_{e}\right\|<e$; hence $x \in I$.

The theorem now follows from Corollaries 2.2.1 and 2.6.1, and Theorem 2.7.

It is easily seen that the first part of Wiener's general Tauberian theorem [37, Theorem 4] and Pitt's Theorem 2 [29] follow from the case $B=A$; and that the second part of Wiener's theorem [37, Theorem 5] results from setting $B=M_{1}$ (see Wiener $\left[37\right.$, p. 73] for a definition of $M_{1}$ ), and defining 


$$
\|f(\cdot)\|=\sup _{-\infty<y<\infty} \sum_{n=-\infty}^{\infty} \max _{0 \leqq x \leqq 1}|f(x+y+n)|\left({ }^{26}\right) .
$$

2. The span of translations of elements of function spaces on groups.

Definition 3.1. A (right, left) translation of a function $f(x)$ on a group $G$ is a function of the form $(f(x a), f(a x))$, where a is a fixed element of $G$.

The present section investigates the span of the translations of elements of certain kinds of function spaces, including the Lebesgue spaces with respect to Haar measure, on LC groups. Wiener's theorems [36] giving NASCs that the span constitute (the entire spaces) either $L$ or $L_{2}$ is extended to groups which are either LC abelian or compact. In the case of $L_{p}$ spaces on LC abelian groups it is shown that conditions analogous to those for $L$ and $L_{2}$ (requiring that the Fourier transform not vanish) are sufficient when $p=2$ and necessary when $1<p<2$, but, in case the group is the additive group of the real numbers, not sufficient when $1<p<2$, settling a well known question raised by Wiener. On the other hand, for $L_{p}$ spaces on the additive group of the real numbers a condition is given which is sufficient but probably not necessary, and which extends results of Agnew's. In addition to the foregoing results, which concern the conditions under which the entire space is spanned, the intuitional relationship between the span and the spectrum of a set of functions is confirmed by showing that the spectrum is a function of the span, and that the span includes all functions whose spectrum is interior to (in the topology of Part II) the spectrum of the set of spanning functions.

The main physical significance of the study of spanning, in the opinion of the author, is that the span of the translations of functions of time is a major invariant of the functions, when they are related to a physical system whose characteristics are independent of absolute time, and in which linear combinations of the functions is meaningful; and that the analogous is true in the case of physical systems whose characteristics are invariant under an associated LC group.

Our chief tool is the spectral resolution for closed ideals in group algebras. First it is shown that the span of the translations of elements of a space satisfying conditions (a)-(c) of Theorem 3.1 is a closed ideal in an algebra built on the space; the spectral resolution is then applied as in the proof of Theorem 3.1. In the case of Lebesgue spaces of exponent greater than unity, Lemma 3.3.1 is important.

THEOREM 3.2. Let $G$ be a group which is either LC abelian or compact, and

${ }^{(26)}$ Other theorems of a similar character can be obtained by the same method. Essentially, the method consists of, first, showing that the set of functions satisfying the conclusion of the theorem is an ideal (usually a matter of straightforward verification), and second, applying the ideal spectral resolution, for example, to obtain a general theorem including Theorem $1 \mathrm{~B}$ of the cited paper by Pitt; $\Omega$ is put equal to the entire conjugate space and the spectral resolution for a not necessarily closed ideal applied. 
$B$ a dense linear subspace of the group algebra $A$ of $G$ and $a$ Banach space with respect to a norm $\|\cdot\|$ (not necessarily the same as the norm on $A$ ). Assume that $B$ satisfies the following three conditions:

(a) $f(\cdot) \in B$ implies that $f_{a}(\cdot)$ and $f^{a}(\cdot)$ are elements of $B$ for every $a \in G$, where $f_{a}(x)=f\left(a^{-1} x\right)$ and $f^{a}(x)=f\left(x a^{-1}\right)$.

(b) The mappings $a \rightarrow f_{a}$ and $a \rightarrow f^{a}$ on $G$ to $B$ are continuous for $f \in B$, and $\left\|f_{a}\right\|=\left\|f^{a}\right\|=\|f\|$.

(c) If $\|f(\cdot, n)\| \rightarrow 0$, then $\int_{G}|f(x, n)| d x \rightarrow 0$.

Then the $B$-span of the translations of elements of a set $K$ of $B$ contains all elements whose $A$-spectrum is interior to that of $K$.

Lemma 3.2.1. The span of the (left or right) translations of elements of $K$ is the closure of the set $T$ of all products ( $g f$ or $f g$ ) with $g \in A, f \in K$.

It is plain from the theory of the $\mathrm{BDH}$ integral that the integral ( $\int_{G} f_{a} g(a) d a$ or $\left.\int_{G} f^{a} g(a) d a\right)$ exists and is in the span of (left or right) translations of elements of $K$, if $f \in K$ and $g(\cdot) \in A$. On the other hand, from the closed-graph theorem, $\int_{G} f_{a} g(a) d a=g f$ and $\int_{G} f^{a} g(a) d a=f g$. That $\left(f_{a}\right.$ or $\left.f^{a}\right)$ is in the closure of $T$ is seen by putting $g(x)=(m(E))^{-1} h(x)$, where $h(\cdot)$ is the characteristic function of the set $E, E$ being a neighborhood of $a$, and $m(\cdot)$ being Haar measure, and noting that the $B$-limit of $\left(g f\right.$ or $f g$ ) is $\left(f_{a}\right.$ or $\left.f^{a}\right)$, because ( $g f$ or $f g$ ) is in any closed convex set containing $\left[f_{b} \mid b \in E\right]$ or $\left[f^{b} \mid b \in E\right]$.

Lemma 3.2.1 shows that the span $S$ of the translations of elements of $K$ is closed in $B$ and an ideal in $A$. It is easy to verify that the $A$-closure $\bar{S}$ of $S$ is an ideal in $A$ and that $\bar{S} \cap B=S$ (compare the proof of a similar fact in Theorem 3.1). The theorem now follows from Corollaries 2.2.1 and 2.6.1.

CoROLLARY 3.2.1. A NASC that a subset $K$ of the group algebra $A$ of a group, which is either LC abelian or compact, span $A$ is that $K$ be contained in no regular maximal ideal.

Setting $B=A$ in Theorem 3.2 and noting that the spectrum of $A$ is interior to that of $K$ if $K$ is contained in no regular maximal ideal yields the corollary.

CoROllaRY 3.2.2. Let $G$ be compact and $B$ either one of the spaces $L_{p}(G)$, $1 \leqq p<\infty$, or the space of continuous functions on $G$ with the norm $\|f(\cdot)\|$ $=\sup _{x \in G}|f(x)|$. Let $K$ be an arbitrary subset of $B$ and put $\Delta$ for the set of all continuous irreducible representations of $G, r(\cdot)$, such that $\int_{G} k(x) r(x) d x=0$, for all elements of $k(\cdot)$ of $K$. Then the B-span of the translations of the elements of $K$ consists of all $f(\cdot) \in B$ such that

for every $r(\cdot) \in \Delta$.

$$
\int_{G} f(x) r(x) d x=0
$$

It is easy to verify that $B$ satisfies the hypothesis of Theorem 3.2. Utilizing Theorems 1.9 and 2.4 , the corollary follows. 
THEOREM 3.3. Let $G$ be a $L C$ abelian group and $K$ a subset of $L_{p}(G)$ such that the $L_{p}$-Fourier transform of every element of $K$ exists. (If $p>2$, the $L_{p}$-Fourier transform of an element $f$ of $L_{p}$ is said to exist if $f$ is the $L_{q}$-Fourier transform of an element of $L_{q}$ where $p^{-1}+q^{-1}=1$.) In order that the translations of elements of $K$ span $L_{p}$, it is sufficient when $2 \leqq p<\infty$, and in case $K$ is countable, necessary when $1<p \leqq 2$, that: $\left(^{*}\right)$ for every set $S^{*}$ in the continuous character group $G^{*}$ of $G$ which is a countable sum of compact sets, there exists a subset of $K$ that is finite or countable, and such that the common part of $S^{*}$ and the zeros of the $L_{p}$-Fourier transforms of the elements of the subset is a set of Haar measure zero.

LEMma 3.3.1. A NASC that the translations of the elements of a set $K$ in $L_{p}$ span $L_{p}$ is that the following be true: if $g(\cdot) \in L_{q}$, where $p^{-1}+q^{-1}=1$, and $\int_{G} f\left(x y^{-1}\right)(g(y))^{c} d y=0$ for all $f(\cdot) \in K$, then $g(\cdot)=0$.

By a lemma of Banach $\left({ }^{27}\right)$, the translations of $f(\cdot) \operatorname{span} L_{p}$ if and only if no continuous linear functional vanishes on all translations except the zero functional. By the extension of Riesz' representation $\left({ }^{28}\right)$ theorem to general Lebesgue spaces a continuous linear functional on $L_{p}, \lambda$, has the form

$$
\lambda f(\cdot)=\int_{G} f(x)(g(x))^{\bullet} d x,
$$

where $g(\cdot)$ is a particular element of $L_{q}$. The proof is completed by combining these facts in straightforward fashion.

Consider first the case $p \geqq 2$. An indirect proof is given: suppose that $\left(^{*}\right)$ is satisfied but that the translations of the elements of $K$ do not span $L_{p}$. By Lemma 3.3.1 there exists a nonzero element $g$ of $L_{q}$ such that $f g^{c}=0$ for $f \in K, g^{c}$ being the complex conjugate of $g$. Since $1<q \leqq 2$, the $L_{q}$-Fourier transform of $g, G(\cdot)$, exists. By hypothesis the $L_{p}$-Fourier transform of $f$, $F(\cdot)$, exists, if $f \in K$. Hence the Parseval formula for Fourier transforms $[35$, p. 118] applies, in the following manner:

$$
f g^{c}(x)=\int_{G^{*}} x^{*} x F\left(x^{*}\right)\left(G\left(x^{*}\right)\right)^{\bullet} d x^{*} .
$$

Now $G\left(x^{*}\right)$ is zero except on a countable sum of compact sets, because $G(\cdot)$ is an element of $L_{p}$. Noting that $F\left(x^{*}\right)\left(G\left(x^{*}\right)\right)^{c}$ is an element of $L_{1}$, it follows from uniqueness theorems for Fourier transforms and the validity of $\left(^{*}\right)$ that $g(\cdot)=0$, a contradiction.

Now suppose $1<p \leqq 2$. Again an indirect proof is given: let $K$ be a countable set of elements whose translations span $L_{p}$ and whose $L_{p}$-Fourier transforms vanish on the measurable set $S^{*}$ of positive measure. Plainly there

${ }^{(27)}$ S. Banach, Théorie des opérations linéaires, Warsaw, 1932, p. 57.

(28) F. Riesz, Untersuchungen uber Systeme integrierbarer Funktionen, Math. Ann. vol. 69 (1910) p. 475. (The extension applies to $L_{p}$ spaces over an arbitrary complete $\sigma$-field of sets with a completely additive measure, the entire space being not necessarily an element of the field.) 
exists a measurable set of finite positive measure $E^{*}$ on which the $L_{p}$-Fourier transform of $f$ vanishes for $f \in K$. Putting $H$ for the characteristic function of $E^{*}$, plainly $H \in L_{p}\left(G^{*}\right)$; hence $H$ is the $L_{q}$-Fourier transform of an element of $L_{q}(G), h$. Using the Parseval formula as in the preceding paragraph, it follows that $f h=0$ if $f \in K$, constituting a contradiction, by Lemma 3.3.1.

In case $p=2$ and $K$ is countable, $\left({ }^{*}\right)$ is NAS, plainly. For $K$ a single function and in the case of the additive group of the reals, this has been proved by Wiener [36], our method of proof of sufficiency being simpler but less elementary than that of Wiener. It is natural to ask, as Wiener did [36, p. 93], whether the same theorem is true for other values of $p$, and it has been shown by the author [31] that it is not. For completeness, the specific result is quoted.

THEOREM 3.4. There exists for every $p$ satisfying $1<p<2$ an element of $L_{p}$ on the additive group of the real numbers whose $L_{p}$-Fourier transform is not zero except on a set of Lebesgue measure zero, and whose translations do not span $L_{p}$.

The following theorem indicates that there exists a positive connection between the span of the translations of an element of $L_{p}$ on the additive group of the real numbers and its suitably-defined Fourier transform. Agnew's theorems $[1,2]$ about spans in $L_{p}$ are special cases $\left({ }^{29}\right)$.

THEOREM 3.5. Let $f$ be an element of both $L_{p}$ and $L_{1}$, over the additive group of the real numbers, such that the set of zeros of its $L$-Fourier transform has no limit points, and where $1<p<\infty$. Then the translations of $f \operatorname{span} L_{p}$.

Utilizing the notation of Lemma 2.7.1, it is easy to verify that $\|\lambda(\cdot, s, a)\|_{p}^{p}=O\left(s^{p-1}\right)$ as $s \rightarrow 0,\|\cdot\|_{p}$ designating the $L_{p}$ norm. Now put $B$ for the common part of $L_{1}$ and $L_{p}$, with the norm $\|\cdot\|_{1}+\|\cdot\|_{p}$. Let $g$ be any element of $L_{p}$ and $e$ a real positive number. Simple functions being dense in $L_{p}$, there exists $g_{\theta} \in B$ such that $\left\|g-g_{e}\right\|_{p}<e$. Putting $G_{\theta}(\cdot)$ for the $L_{1}$-Fourier transform of $g_{e}$, and $G_{e, \alpha}(t)=G_{e}(t) \prod_{m=1}^{\infty}\left(1-\Lambda\left(t, C_{n}, z_{m}\right)\right)$, where $\alpha$ is the set of positive numbers $\left\{C_{n}\right\}$ and $\left\{z_{n}\right\}$ is the set of zeros of the $L_{1}$-Fourier transform of $f$, and noting that

$$
\left\|x-\prod_{n=1}^{\infty}\left(I+T_{n}\right) x\right\| \leqq \exp \left(\sum_{n=1}^{\infty}\left\|T_{n}\right\|-1\right)\|x\|,
$$

where $x$ is an element of an (arbitrary) Banach space and the $T_{n}$ are bounded and mutually commuting operators on that space to a subset of that space (with a different norm), it follows that there exists a set $\left\{C_{n}\right\}$ such that $G_{e, \alpha}(\cdot)$ is the $L_{p}$-Fourier transform of an element of $L_{p}, g_{e, \alpha}$, with $\left\|g_{e, \alpha}-g_{e}\right\|_{p}<e$. It follows that $\left\|g-g_{e, \alpha}\right\|_{p}<2 e$. Plainly, $B$ satisfies the hypothesis of Theorem 3.2. By Lemma 3.2.1 and the remark at the end of the proof of this lemma,

${ }^{(29)}$ Agnew's proofs include direct methods of approximation. 
$g_{e, \alpha}$ is in the $B$-span (and hence in the $L_{p}$-span) of the translations of $f$, completing the proof.

3. Analytic functions on group algebras. In this section theorems are proved about the existence of analytic functions on the group algebra of a group which is either compact or LC abelian, and on an algebra associated with a LC abelian group obtained by combining with the group algebra the algebra of convolutions of purely discontinuous completely additive set functions. Our results for the case of an abelian group constitute an extension of theorems published by Gelfand and Raikov [15] (the present work was done without knowledge of their work however), and include the basic case of a theorem obtained independently by Beurling [4] and jointly by Cameron and Wiener [6] $\left.{ }^{(30}\right)$ about analytic functions on an algebra associated with the additive group of the real numbers.

Continuous functions are not generally definable on group algebras. It is easy to see that otherwise every sequence tending to zero would be the sequence of Fourier coefficients of an integrable function.

THEOREM 3.6. Let $f$ be an element of the group algebra $A$ of a compact group and $\phi(\cdot)$ a function that is analytic and single-valued on a connected open set containing zero and the closure of the set of all proper values of matrices into which $f$ is mapped by homomorphisms of $A$ with kernels which are regular and maximal, and such that $\phi(0)=0$. Suppose either that $f$ commutes with $f^{*}$ or that $\phi(\cdot)$ is a meromorphic function. Then there exists an element $g$ in $A$ such that for every regular maximal ideal $M$ in the group algebra,

$$
g_{M}=\phi\left(f_{M}\right),
$$

where $f_{M}$ and $g_{M}$ are matrices into which $f$ and $g$ are mapped by a homomorphism of $A$ with kernel $M, f_{M}$ being a normal matrix if $f$ commutes with $f^{*}, \phi\left(f_{M}\right)$ being defined in a fashion similar to that for diagonal matrices, and so as to commute with similarities ${ }^{\left({ }^{1}\right)}$.

Lemma 3.6.1. In order that an element of a CC semi-simple algebra have an adverse it is sufficient that it have an adverse modulo every regular maximal ideal in the algebra.

$\left.{ }^{(30}\right)$ The results of these authors are formally somewhat more general than ours (for the case of the reals). However, it is easy to verify that the present methods could be utilized to obtain theorems of the type that these authors prove, with the (possible) exception of Cameron and Wiener's results about the existence of multi-valued analytic functions of Fourier-Stieltjes transforms.

${ }^{(31)}$ We are indebted to $\mathrm{N}$. Dunford for pointing out that the theorem remains true in the more general case that $\phi(\cdot)$ is analytic and single-valued on each of a finite collection of connected open sets, the value of this type of analytic function of a matrix being defined by the Cauchy integral formula. See N. Dunford, Spectral theory. I, Trans. Amer. Math. Soc. vol. 54 (1943) pp. 185-217, esp. pp. 192-193. 
Put $A$ for the algebra and $U_{x}$ for the left regular representation of the element $x$ of $A$, that is, the operator taking $y$ into $x y$, where $y$ is a general element of $A$. By the Riesz-Hildebrandt theory of CC operators, either $I-U_{x}$ has a bounded inverse, $I$ being the identity operator, or there exists a nonzero $y \in A$ such that $\left(I-U_{x}\right) y=0$. Reducing this last relation modulo a regular maximal ideal $M$ shows that $y-x y \in M$. Multiplying by an element that is an adverse of $x$ modulo $M$ shows that $y \in M ; A$ being semi-simple, this implies $y=0$. Hence $I-U_{x}$ has a bounded inverse. Putting $u=-\left(I-U_{x}\right)^{-1} x$, it is easy to verify that $x u=u+x$, that is, $u$ is a right adverse of $x$. Utilizing the right regular representation of $x$ in similar fashion, it follows that $x$ has, also, a left adverse, and therefore $x$ has an adverse.

Considering first the case in which $f$ is normal, it is easy to show that $f_{M}^{*}$ is the hermitian conjugate of $f_{M}$, in case the group representation corresponding to the homomorphism is unitary. Every continuous finite-dimensional representation of a compact group being equivalent to a unitary representation, it follows that $f_{M}$ is normal if $f$ commutes with $f^{*}$. Put $D$ for the range of the proper values of $f_{M}$ for all regular maximal ideals $M$, and $E$ for the connected open set containing zero and $\bar{D}$ on which $\phi(\cdot)$ is single-valued and analytic. Let $F$ be an open set such that $\bar{F} \subset E, \bar{D} \subset F$, and whose boundary consists of a finite number of polygons. Writing $B$ for the suitably oriented boundary of $F$, it follows easily from Cauchy's theorem that:

$$
\phi(\alpha)=-\frac{1}{2 \pi i} \int_{B} \frac{\phi(\beta)}{\beta} \frac{\alpha}{\alpha-\beta} d \beta,
$$

for $\alpha \in D$. It is plain that the adverse of $\beta^{-1} f_{M}$ exists for every $\beta \in B$ and regular maximal ideal $M$. Hence $\left(\beta^{-1} f\right)^{A}$ exists. It is easy to verify that if $x$ is an element of a $B$-algebra such that $x^{A}$ exists, then there exists a neighborhood $N$ of $x$ such that $u^{A}$ exists for $u \in N$ and the mapping $u \rightarrow u^{A}$ is continuous. Hence the mapping $\beta \rightarrow\left(\beta^{-1} f\right)^{A}$ on $B$ to $A$ is continuous; since $B$ is compact, the range of this mapping is bounded. Hence the following BDH integral exists:

$$
-\frac{1}{2 \pi i} \int_{B} \frac{\phi(\beta)}{\beta}\left(\beta^{-1} f\right)^{\Lambda} d \beta .
$$

Putting $g$ for this integral, it is clear that $g_{M}=\phi\left(f_{M}\right)$ (the mapping $h \rightarrow h_{M}$ being linear and bounded).

Now suppose that $\phi(\cdot)$ is meromorphic, and that $f$ is not necessarily normal. We can plainly set $\phi(z)=\phi_{1}(z) \phi_{2}(z)$, where $\phi_{1}(z)$ is rational, and regular at every point at which $\phi(z)$ is regular, and where $\phi_{2}(z)$ has a convergent power series expansion in the circle $|z| \leqq\|f\|+1$, and $\phi_{2}(0)=0$. Clearly, $\phi_{2}(f) \in A$. Now Lemma 3.6.1 shows that the theorem is valid for $\phi(z)=z(z-1)^{-1}$, and it follows easily that it is valid for $\phi(z)=z(z-\alpha)^{-1}, \alpha$ being any complex number. It is plain that $\phi_{1}(z)$ has the form $p(z) \prod_{i=1}^{n}\left(z-\alpha_{i}\right)^{-1}$, 
where $p(z)$ is a polynomial and $p\left(\alpha_{i}\right) \neq 0$ for every $i$. Noting that $(z-\alpha)^{-1}$ $=z(z-\alpha)^{-1}-1$, and observing that $\psi(f) \in A$ if $\psi(\cdot)$ has the form $z \prod_{i \in S}\left(z-\alpha_{i}\right)$, $S$ being any set of indices, it follows that $\phi(f) \in A\left({ }^{32}\right)$.

Theorem 3.7. Let $G$ be a $L C$ abelian group. Put $R$ for the set of all complexvalued completely additive set functions on $G$ of the form $\alpha(\cdot)+\beta(\cdot)$, where $\alpha(\cdot)$ is absolutely continuous with respect to Haar measure and $\beta(\cdot)$ has the form

$$
\beta(E)=\sum_{x \in E} b(x),
$$

$b(x)$ vanishing except on a countable set of points. Let $\gamma(\cdot)$ be an element of $R$ and suppose that $\phi(\cdot)$ is a function that is analytic and single-valued on a connected open set containing the closure of the range of the Fourier-Stieltjes transform of $\gamma(\cdot), \Gamma(\cdot)\left(\Gamma\left(x^{*}\right)=\int_{G} x^{*} x d \gamma\left(E_{x}\right)\right)$, over the range $G^{*}$, the group of continuous characters of $G$. Then there exists an element $\delta(\cdot)$ in $R$ whose FourierStieltjes transform $\Delta(\cdot)$ satisfies:

$$
\phi\left(\Gamma\left(x^{*}\right)\right)=\Delta\left(x^{*}\right),
$$$$
x^{*} \in G^{*} \text {. }
$$

LEMma 3.7.1. Let $f(\cdot)$ be a continuous almost periodic function on the not compact $L C$ abelian group $G$. Then if $C$ is any compact subset of $G$,

$$
\inf _{x \in G}|f(x)|=\inf _{x \in G-C}|f(x)| .
$$

The proof is indirect. Suppose that $C$ is compact, $e$ is a positive number, and $\inf _{x \in G-C}|f(x)| \geqq \inf _{x \in G}|f(x)|+e$. Because $G$ is not compact there exists a sequence $\left\{a_{i}\right\}$ in $G$ such that $a_{i} C$ and $a_{j} C$ are disjoint, when $i \neq j$. It is easy to verify that $\left|f\left(a_{i} x\right)-f\left(a_{j} x\right)\right| \geqq e$, when $i \neq j$. Hence $f(\cdot)$ is not almost periodic, a contradiction.

Lemma 3.7.2. Let $\gamma$ be an element of $R$ such that $\inf _{x^{*} \in G^{*}}\left|\Gamma\left(x^{*}\right)\right|>0$, where $\Gamma(\cdot)$ is the Fourier-Stieltjes transform of $\gamma(\cdot)$. Then $\gamma$ has an inverse with respect to convolution in $R$.

Consider first the case of a discrete group. In that case, $R$ regarded as an algebra, multiplication being defined as convolution, is obviously isomorphic with the group algebra. An element $\boldsymbol{\gamma}$ satisfying the hypothesis is clearly contained in no maximal ideal, and hence the ideal generated by $\gamma$ is $R$, showing that $\boldsymbol{\gamma}$ has an inverse.

(2a) An analogue of Theorem 3.6 for a general LC group can readily be obtained by: (a) utilizing in place of the class $\Gamma$ of regular maximal ideals the class $\Phi$ of ideals which are either of the form $[x \mid A x \subset R]$ or of the form $[x \mid x A \subset L]$, where $R$ and $L$ are respectively regular maximal right and left ideals, (b) restricting the analytic function to be meromorphic. (It is easy to verify that $\Gamma=\Phi$ in the case of a group which is either LC abelian or compact.) To see this, observe first that an element of a group algebra has an adverse if and only if it has an adverse modulo every ideal in $\Phi$ (by Theorem 1.3), which proves the analogue for the case of the function $\phi(z)=z(z-1)^{-1}$. The case of a general meromorphic function (it is clear how to define such a function on a $B$-algebra) then follows just as in the case of a compact group. 
Assume now that $G$ is not discrete; then $G^{*}$ is not compact. Let $\alpha$ and $\beta$ be the absolutely continuous and purely discontinuous constituents of $\gamma$, and $A(\cdot)$ and $B(\cdot)$ their Fourier-Stieltjes transforms respectively. By the generalized Riemann-Lebesgue lemma $\left[35\right.$, p. 116], $\left[x^{*}|| A\left(x^{*}\right) \mid \geqq e\right]$ is compact if $e$ is a positive number; hence $\inf _{x^{*} \in G^{*}}\left|B\left(x^{*}\right)\right|>0$, applying Lemma 3.7.1. If we put $G^{\prime}$ for the group $G$ considered as a discrete group, it is plain that $G^{*}$ is a dense subgroup of $G^{* *}$. If we define

$$
B^{\prime}\left(x^{\prime *}\right)=\int_{G} x^{\prime *} x d \beta\left(E_{x}\right)
$$

clearly $B^{\prime}(\cdot)$ is continuous on $G^{\prime *}$; hence $\inf _{x^{\prime \bullet} \in G^{\prime \bullet}}\left|B^{\prime}\left(x^{*}\right)\right|>0$. It follows, by the result of the preceding paragraph, that $\gamma$ has an inverse in $R$.

It is easy to verify that $-\alpha \beta^{-1}$ is absolutely continuous. Utilizing the isomorphism between the group algebra of $G$ and the subalgebra $A^{\prime}$ of $A$ consisting of elements that are absolutely continuous, it follows that $-\alpha \beta^{-1}$ has an adverse modulo every regular maximal ideal in $A^{\prime}$, and hence an adverse in $R$. Setting $\sigma=\beta^{-1}-\beta^{-1}\left(-\alpha \beta^{-1}\right)^{A}$, it is easy to show that $\sigma(\alpha+\beta)$ is the identity, completing the proof.

Lemma 3.7.3. Let $T$ be a commutative Banach algebra with an identity $e$, and put $\Lambda$ for a family of maximal ideals in $T$ with the property that $\inf _{M \in \Delta}|x(M)|>0$ implies that $x$ has an inverse in $T, x(M)$ being a complex number defined by the congruence $x \equiv x(M) e$ modulo $M$. Let $t$ be an element of $T$, and suppose that $\phi(\cdot)$ is a function that is analytic and single-valued on a connected open set containing the closure of the range of $t(M), M$ varying over the domain $\Delta$. Then there exists an element $u$ in $T$ such that

for $M \in \Delta$.

$$
u(M)=\phi(t(M))
$$

The proof is the same as the proof of the corresponding fact for Theorem 3.6 except that inverses are used instead of adverses.

The proof of the theorem may now easily be concluded by utilizing Lemmas 3.7.2 and 3.7.3.

THEOREM 3.8. Let $f$ be an element of the group algebra of the locally compact abelian group $G$. Suppose that $\phi(\cdot)$ is analytic and single-valued on a connected open set containing the closure of the range $D$ of $F\left(x^{*}\right)$, where $F(\cdot)$ is the Fourier transform of $f$ and $x^{*}$ ranges over the subset $N^{*}$ of the group $G^{*}$ of continuous characters of $G^{*}$, and suppose also that if zero is in $\bar{D}$, then $\phi(0)=0$. Then there exists an element of $A$ whose Fourier transform $H(\cdot)$ satisfies $H\left(x^{*}\right)=\phi\left(F\left(x^{*}\right)\right)$.

Consider first the case in which $\left(N^{*}\right)^{\circ}$ is compact. Put $I$ for the ideal in $A$ consisting of elements whose Fourier transforms vanish on $N^{*}$. Then $A / I$ has an identity, by Theorem 2.5. Setting $\Delta$ for the family of regular maximal 
ideals in $A$ corresponding to $N^{*}$, it is clear that every maximal ideal in $A / I$ has the form $M / I$, with $M \in \bar{\Delta}$; on the other hand, $A / M \sim(A / I) /(M / I)$, by the isomorphism law for ideals. Hence if $u \in A$ and $M \in \bar{\Delta}, u(M)=(u+I)(M / I)$, showing that if $\inf _{M^{\prime} \in \Delta_{I}}\left|(u+I)\left(M^{\prime}\right)\right|>0$, where $\Delta_{I}$ is the family of ideals of the form $M / I$ with $M \in \Delta$, then $\inf _{M \in \Delta}|u(M)|>0$. By virtue of the continuity of $u(\cdot)$, the last equation implies $\inf _{M \in \bar{\Delta}}|u(M)|>0$; hence $\inf _{M \in \Gamma_{I}}|(u+I)(M)|>0$, where $\Gamma_{I}$ is the spectrum of $A / I$. Plainly, the conditions of Lemma 3.7.3 are satisfied, setting $T=A / I$ and $\Lambda=\Gamma_{I}$, and the validity of the theorem in this case follows.

Suppose now that $\bar{N}^{*}$ is not compact. Then zero is in $\bar{D}$, by the generalized Riemann-Lebesgue lemma, and the proof is concluded by combining the discussion of the case in which $\left(N^{*}\right)^{c}$ is compact with the analogue to Lemma 3.7.3 for the case of an algebra without an identity, this analogue being proved in the proof of Theorem 3.6.

4. A new approach to the theory of almost periodic functions on groups. The principal results of von Neumann's theory of numerical-valued almost periodic (AP) functions on groups are frequently considered to be the approximation theorem (that is, the extension of the Peter-Weyl theorem), the fact that a LC abelian group is maximally $\mathrm{AP}\left({ }^{33}\right)$, and the existence of the mean. The purpose of the present section is to derive the first two of these results from the foregoing theory of group algebras; for completeness the third result is also proved, from the existence of Haar measure on compact groups. This derivation seems worth while partly because of its logical simplicity, the group algebra results following rather directly from the existence of Haar measure and the spectral theorem, partly because the proofs are relatively short, and partly because it seems aesthetically satisfying that the approximation theorem can be formulated in highly algebraic terms, as the semi-simplicity of the group algebra of a compact group.

Our starting point is the fact that the space of continuous AP functions on a topological group is essentially equivalent to the space of continuous functions on an appropriate compact group. This fact was first derived by Tannaka from von Neumann's results (it had been proved earlier by Pontryagin in the case of the additive group of the real numbers). It is proved here directly from the definition of almost periodicity, utilizing a topological device introduced by Stepanoff and Tychonoff [32] and later used by Weil and van Kampen. These authors investigated a single AP function by retopologizing the group in a manner depending on the function, and completing the resulting metric group. In the present treatment all AP functions are simultaneously utilized, the retopologized group being not necessarily metric, but completable as a group, by the theory of uniform spaces $\left({ }^{34}\right)$-a de-

(23) A maximally AP group is one on which there exists for every two points of the group a continuous AP function which assumes different values at the points (according to [27]).

(34) See A. Weil, Sur les espaces a structure uniforme et sur la topologie generale, Actualites Scientifiques et Industrielles, no. 551, Paris, 1938. 
velopment which was not in existence at the time of those authors' investigations.

The approximation theorem follows from the semi-simplicity of the group algebra of the compact group mentioned in the previous paragraph. The maximal almost periodicity of LC abelian groups is implied by (but does not imply) the semi-simplicity of the group algebra of a LC abelian group.

THEOREM 3.9. Let $R$ be the set of all complex-valued AP functions on the topological group $G$. Put $K$ for the class of all elements of $G$ on which every function in $R$ assumes the same value as on the unit. Then $K$ is a closed normal subgroup of $G$, and there exists a compact group $\bar{G}$ such that:

(a) There exists a continuous uniform homomorphism $\phi(\cdot)$ on the topological group $G / K$ to a dense subgroup of $\bar{G}$.

(b) There exists a biuniform exhaustive linear mapping $T$ on $R$ to the family of complex-valued continuous functions on $\bar{G}$ such that, for $f(\cdot) \in R$ and $x \in G$, $f(x)=(T f)(\phi(x))$.

It is easy to verify that $K$ is a closed normal subgroup. Putting $G / K=H, \psi(\cdot)$ for the (continuous) homomorphism on $G$ to $H$, and $S$ for the class of all complex-valued continuous AP functions on $H$, it is straight-forward to show that $h(\cdot) \in S$ if and only if $h(\psi(x)) \in R$. Thus $H$ is maximally AP.

The underlying group of $H$ will now be retopologized to form a uniform topological group $H^{*}$. A general neighborhood family $N(h), h \in H^{*}$, is defined to be the set of all elements $k$ in $H^{*}$ such that:

$$
\sup _{x, y \in H^{*}}\left|f_{i}(x k y)-f_{i}(x h y)\right|<e \quad(i=1, \cdots, n),
$$

where $\left\{f_{\mathbf{1}}(\cdot), \cdots, f_{n}(\cdot)\right\}$ is a general finite subset of $S$ and $e$ is a general positive real number. It is easy to verify that $H^{*}$ is a uniform topological group. Noting that if $f(\cdot) \in S$, then $f\left(x y^{-1}\right)$ is right AP on $H^{*} \times H^{*}$, we see from the definition of almost periodicity that $H^{*}$ is totally bounded. On the other hand, if $g(\cdot)$ is uniformly continuous on $H^{*}$, it is clear that the mappings $a \rightarrow g_{a}(\cdot)$ and $a \rightarrow g^{a}(\cdot)$, where $g_{a}(x)=g(x a)$ and $g^{a}(x)=g(a x)$, are continuous on $H^{*}$ to the space of complex-valued functions with the distance between two functions defined to be the L.U.B. of their difference. Hence the ranges $\left[g^{a}(\cdot) \mid a \in H^{*}\right]$ and $\left[g_{a}(\cdot) \mid a \in H^{*}\right]$ are totally bounded, that is, $g(\cdot)$ is AP; plainly, then, $g(\cdot) \in S$. Since neighborhoods of the identity are invariant under transformation by elements of the group, $H^{*}$ is imbeddable in a complete group; if we call this group $\bar{G}$, it is plain that the conclusions of the theorems are valid.

THEOREM 3.10. A continuous almost periodic function on a topological group can be uniformly approximated arbitrarily closely by linear combinations of coordinates of bounded, continuous, irreducible, finite-dimensional representations. 
Adopt the notation of the preceding theorem and put $f$ for a general element of $R$. The main fact used in the proof is that $T f$ can be approximated in the group algebra $A$ of $\bar{G}$ by linear combinations of coordinates of bounded, continuous, irreducible, finite-dimensional (BCIF) representations of $\bar{G}$, this being proved by an indirect argument. Assume that $T f$ is not in the $A$-span of the coordinates of BCIF representations of $\bar{G}$. Then there exists a continuous linear functional on $A$ which vanishes on all such coordinates and is not zero (by a lemma of Banach's). Utilizing Riesz' representation theorem for continuous linear functionals on an $L_{1}$-space, there exists a measurable bounded function $g(\cdot)$ on $\bar{G}$, which does not vanish almost everywhere, such that $\int_{G} g(x) r(x) d x=0$ for a general coordinate $r(\cdot)$ of a general BCIF representation of $\bar{G}$. On the other hand, it is obvious that $g(\cdot) \in A$. Combining this fact with Theorems 1.7 and 1.9 , we see that $g(\cdot)=0$, a contradiction.

The proof is concluded by an approximation argument. Let $h$ be an arbitrary complex-valued continuous function on $\bar{G}$, and define $\|h\|_{0}$ $=\sup _{x \in \bar{G}}|h(x)|$ for such a function. Putting $e$ for a general positive number, let $f$ be an element of $A$ such that $\|h-f h\|_{c}<e$, and let $g$ be a linear combination of coordinates of BCIF representations of $\bar{G}$ such that $\|f-g\|_{A}<e\left(\|h\|_{c}\right)^{-1}$. Noting that $\|p q\|_{c} \leqq\|p\|_{A}\|g\|_{c}$, we see that $\|h-g h\|_{c}<2 e$, concluding the proof, because $g h$ is expressible as a finite linear combination of coordinates of BCIF representations of $\bar{G}$, and every such representation of $\bar{G}$ corresponds by Theorem 3.8 to a BCIF representation of $G$.

TheOREM 3.11. A LC abelian group is maximally almost periodic.

Employing an indirect proof, utilize the notation of Theorem 3.8 and assume that all functions in $A$ take on the same value at the point $a$ of $G$ as at the identity of $G$, which is not $a$. Obviously a character of $G$ is AP, and therefore

$$
\int_{G} x^{*} x[f(x)-f(a x)] d x=0,
$$

where $f(\cdot)$ is a general element of $A$. By Theorem 1.9 and the fact that the group algebra of a LC abelian group is semi-simple, $f(x)=f(a x)$ almost everywhere, showing that $a$ is the identity, a contradiction.

THEOREM 3.12. If we employ the notation of Theorem 3.8, the mean value of $a$ general element $f$ of $R$ is $\int_{\bar{G}}(T f) d x$, where $d x$ is Haar measure on $\bar{G}$, the measure of $\bar{G}$ being unity.

If we define $L(f)=\int_{\bar{G}}(T f)(x) d x$ for $f \in R$, it is easy to verify that: (1) $L(\cdot)$ is linear; (2) $L(1)=1$; (3) $L(f) \geqq 0$ if $(f)(x) \geqq 0$ for all $x \in G$; (4) $L\left(f_{a}\right)=L(f)$, where $\left(f_{a}\right)(x)=(f)(x a)$, for $a \in G$. These formal properties determine uniquely the mean, by a theorem of von Neumann's [27, Theorem 7]. 


\section{BIBLIOGRAPHY}

1. R. P. Agnew, Spans in Lebesgue and uniform spaces of translations of step functions, Bull. Amer. Math. Soc. vol. 51 (1945) pp. 229-233.

2. - Spans in Lebesgue and uniform spaces of translations of peak functions, Amer. J. Math. vol. 67 (1945) pp. 431-436.

3. W. Ambrose, Structure theorems for a special class of Banach algebras, Trans. Amer. Math. Soc. vol. 57 (1945) pp. 364-386.

4. A. Beurling, Sur les intégrales de Fourier absolument convergentes et leur application d une transformation fonctionelle, Proceedings of the Ninth Scandinavian Mathematical Congress (1938), Helsingfors, 1939, pp. 345-366.

5. J. W. Calkin, Two-sided ideals and congruences in the ring of bounded operators in Hilbert space, Ann. of Math. vol. 42 (1941) pp. 839-873.

6. R. H. Cameron and N. Wiener. Convergence properties of analytic functions of FourierStieltjes transforms, Trans. Amer. Math. Soc. vol. 46 (1939) pp. 97-109.

7. F. Carlson, Une inegalite, Arkiv för matematik, astronomi och fysik vol. 25B (1934) pp. 1-5.

8. V. Ditkin, On the structure of ideals in certain normed rings (in Russian). Uchenye Zapiski Moskovskogo Gosudarstvennogo Universiteta. Matematiska vol. 30 (1939) pp. 83-130. Reviewed in Mathematical Reviews vol. 1 (1940) p. 336.

9. N. Dunford and B. J. Pettis, Linear operations on summable functions, Trans. Amer. Math. Soc. vol. 47 (1940) pp. 323-392. 432.

10. I. Gelfand, On rormed rings, C. R. (Doklady) Acad. Sci. URSS. vol. 23 (1939) pp. 430-

11. - To the theory of normed rings. II. On absolutely convergent trigonometrical series and integrals, C. R. (Doklady) Acad. Sci. URSS. vol. 25 (1939) pp. 570-572.

12. - Ideale und primare Ideale in normierten Ringen, Rec. Math. (Mat. Sbornik) N. S. vol. 9 (1941) pp. 41-48.

13. - Normierte Ringe, Rec. Math. (Mat. Sbornik) N. S. vol. 9 (1941) pp. 3-24.

14. - Über absolut konvergente trigonometrische Reihen und Integrale, Rec. Math. (Mat. Sbornik) N. S. vol. 9 (1941) pp. 51-66.

15. I. Gelfand and D. Raikov, On the theory of characters of commutative topological groups, C. R. (Doklady) Acad. Sci. URSS. vol. 28 (1940) pp. 195-198.

16. - Irreducible unitary representations of locally compact groups, C. R. (Doklady) Acad. Sci. URSS. vol. 42 (1944) pp. 199-201.

17. I. Gelfand and G. Silov, Über verschiedene Methoden der Einführung der Topologie in die Menge der maximalen Ideale eines normierte Ringes, Rec. Math. (Mat. Sbornik) N. S. vol. 9 (1941) pp. 25-39.

18. E. Hille, On Laplace integrals, Proceedings of the Eighth Scandinavian Mathematical Congress (1934), Lund, 1935, pp. 216-227.

19. E. Hille and J. D. Tamarkin, On the characteristic values of linear integral equations, Acta Math. vol. 57 (1931) pp. 1-76.

20. - Questions of relative inclusion in the domain of Hausdorff means, Proc. Nat. Acad. Sci. U.S.A. vol. 19 (1933) pp. 573-577.

21. - On moment functions, Proc. Nat. Acad. Sci. U.S.A. vol. 19 (1933) pp. 902-908.

22. - On the theory of Laplace integrals, Proc. Nat. Acad. Sci. U.S.A. vol. 19 (1933) pp. 908-912.

23. - On the theory of Laplace integrals, II, Proc. Nat. Acad. Sci. U.S.A. vol. 20 (1934) pp. $140-144$.

24. N. H. McCoy and D. Montgomery, A representation of generalized Boolean rings, Duke Math. J. vol. 3 (1937) pp. 455-459. 
25. F. J. Murray and J. von Neumann, On rings of operators, Ann. of Math. vol. 37 (1936) pp. 116-229.

26. - On rings of operators. IV, Ann. of Math. vol. 44 (1943) pp. 716-808.

27. J. von Neumann, Almost periodic functions in a group. I, Trans. Amer. Math. Soc. vol. 36 (1934) pp. 445-492.

28. F. Peter and H. Weyl, Die Vollständigkeit der primitiven Darstellungen einer geschlossen kontinuirlicher Gruppe, Math. Ann. vol. 97 (1927) pp. 737-755. 112.

29. H. R. Pitt, General Tauberian theorems, II, J. London Math. Soc. vol. 15 (1940) pp. 47-

29a. N. Wiener and H. R. Pitt, On absolutely convergent Fourier-Stieltjes transforms, Duke Math. J. vol. 4 (1938) pp. 420-436.

30. I. E. Segal, The group ring of a locally compact group, I, Proc. Nat. Acad. Sci. U.S.A. vol. 27 (1941) pp. 348-352.

31. - The span of the translations of a function in a Lebesgue space, Proc. Nat. Acad. Sci. U.S.A. vol. 30 (1944) pp. 165-169.

32. W. Stepanoff and A. Tychonoff, Sur les espaces des fonctions presque periodiques, C. R. Acad. Sci. Paris vol. 196 (1933) pp. 1199-1201.

33. M. H. Stone, The theory of representations for Boolean algebras, Trans. Amer. Math. Soc. vol. 40 (1936) pp. 37-111.

34. - Applications of the theory of Boolean rings to general topology, Trans. Amer. Math. Soc. vol. 41 (1937) pp. 375-481.

35. A. Weil, l'Integration dans les groupes topologiques et ses applications, Actualites Scientifiques et Industrielles, no. 869, Paris, 1940.

36. N. Wiener, Tauberian theorems, Ann. of Math. vol. 33 (1932) pp. 1-100.

37. - The Fourier integral and certain of its applications, Cambridge, England, 1933.

YALE UNIVERSITY,

New Haven, Conn.

INSTITUTE FOR ADVANCED STUdy,

Princeton, N. J. 